\title{
Resolutions of Convex Geometries
}

\author{
Domenico Cantone $^{a}$ \\ Department of Mathematics and Computer Science \\ University of Catania \\ Catania, Italy \\ domenico.cantone@unict.it
}

Jean-Paul Doignon ${ }^{b}$

Department of Mathematics Université Libre de Bruxelles Brussels, Belgium

doignon@ulb.ac.be
Alfio Giarlotta

Department of Economics and Business

University of Catania

Catania, Italy

alfio.giarlotta@unict.it

\author{
Stephen Watson \\ Department of Mathematics and Statistics \\ York University \\ Toronto, Canada \\ watson@mathstat. yorku.ca
}

Submitted: Mar 23, 2021; Accepted: Sep 25, 2021; Published: Nov 19, 2021

(C) The authors. Released under the CC BY-ND license (International 4.0).

\begin{abstract}
Convex geometries (Edelman and Jamison, 1985) are finite combinatorial structures dual to union-closed antimatroids or learning spaces. We define an operation of resolution for convex geometries, which replaces each element of a base convex geometry by a fiber convex geometry. Contrary to what happens for similar constructions - compounds of hypergraphs, as in Chein, Habib and Maurer (1981), and compositions of set systems, as in Möhring and Radermacher (1984)—, resolutions of convex geometries always yield a convex geometry.

We investigate resolutions of special convex geometries: ordinal and affine. A resolution of ordinal convex geometries is again ordinal, but a resolution of affine convex geometries may fail to be affine. A notion of primitivity, which generalize the corresponding notion for posets, arises from resolutions: a convex geometry is primitive if it is not a resolution of smaller ones. We obtain a characterization of affine convex geometries that are primitive, and compute the number of primitive convex geometries on at most four elements. Several open problems are listed.
\end{abstract}

Mathematics Subject Classifications: 05B25, 06A07, 52A01

\footnotetext{
${ }^{a}$ In honour of Mario Gionfriddo, for his academic career and recent emeritus professorship.

${ }^{b}$ Corresponding author.
} 


\section{Introduction}

Convex geometries, named after Edelman and Jamison (1985), are finite mathematical structures that capture combinatorial features of convexity from various settings. A 'resolution' of convex geometries, as we define it here, is a procedure that builds a new convex geometry from given ones. Intuitively, this procedure replaces each element of a given 'base' convex geometry by another convex geometry, called a 'fiber', and consistently defines a new family of convex sets on the union of the fibers.

Resolutions of convex geometries are related to a similar construction in the field of choice theory, namely 'resolutions of choice spaces', ${ }^{1}$ recently introduced by Cantone, Giarlotta and Watson (2021). This is hardly surprising in view of an enlightening result of Koshevoy (1999), who shows that there is a one-to-one correspondence between convex geometries and special choice spaces, called 'path independent' (see also Johnson and Dean, 1996 and Johnson and Dean, 2001, who independently obtained the same result). Our construction is also reminiscent of the 'compounds of hypergraphs' (Chein, Habib, and Maurer, 1981) or 'compositions of set systems' (Möhring and Radermacher, 1984). There is, however, a salient difference between these two constructions: a composition of convex geometries may fail to be a convex geometry, whereas a resolution of convex geometries is always a convex geometry.

In this paper, we examine resolutions of two special types of convex geometries: ordinal and affine. We also investigate 'primitive' convex geometries, that is, convex geometries that cannot be obtained as resolutions of smaller convex geometries. In particular, we show that the notion of a primitive convex geometry generalizes the classical notion of a primitive poset (partially ordered set). We also perform some computations related to 'small' convex geometries, showing that among the 6 convex geometries on three elements only 1 is primitive, and exactly 12 of the 34 geometries on four elements are primitive.

The paper is organized as follows. Section 2 collects preliminary facts on convex geometries. Section 3 introduces resolutions of convex geometries, and compares them to compositions. Here we also relate resolutions to homomorphisms of (semi)lattices attached to convex geometries. ${ }^{2}$ Section 4 deals with resolutions of affine and ordinal convex geometries; here we also investigate which of these geometries are primitive, and provide a characterization of affine convex geometries that are resolvable. Section 5 provides a taxonomy of all primitive convex geometries having at most 4 elements. Section 6 collects several questions and open problems.

\section{Background on Convex Geometries}

In this section we provide the basic notions and tools for our analysis.

\footnotetext{
${ }^{1} \mathrm{~A}$ choice space is a pair $(X, c)$, where $X$ is a nonempty set, and $c: 2^{X} \rightarrow 2^{X}$ maps each nonempty set $A \subseteq X$ to a nonempty subset $c(A) \subseteq A$ (and the empty set to the empty set).

${ }^{2}$ This part is based on the insightful comments of a referee.
} 


\subsection{Definitions and Examples}

We start with one of the many equivalent definitions of a convex geometry, a notion that is originally due to Edelman and Jamison (1985). Unless otherwise specified, $X$ is a finite nonempty set, which consists of points or elements, depending on the context. As usual, $2^{X}$ denotes the family of all subsets of $X$.

Definition 1. A convex geometry on a finite nonempty set $X$ is a collection $\mathcal{G}$ of subsets of $X$ satisfying the following three axioms:

(G1) $\varnothing \in \mathcal{G}$;

(G2) $\mathcal{G}$ is closed under intersection: if $F$ and $G$ are in $\mathcal{G}$, then $F \cap G$ is in $\mathcal{G}$;

(G3) $\mathcal{G}$ is upgradable: for any $G$ in $\mathcal{G} \backslash\{X\}$, there exists $x$ in $X \backslash G$ such that $G \cup\{x\} \in \mathcal{G}$.

Here $X$ is the ground set of $\mathcal{G}$, and the sets in $\mathcal{G}$ are called convex. We slightly abuse terminology, and also call the pair $(X, \mathcal{G})$ a convex geometry. A convex geometry $(X, \mathcal{G})$ is nontrivial if $|X| \geqslant 2$, and trivial otherwise.

Since $X$ is finite, Axioms (G1) and (G3) of a convex geometry $(X, \mathcal{G})$ imply that $X=\bigcup \mathcal{G} \in \mathcal{G}$. Thus, for any $A \in 2^{X}$, the family of convex sets $G$ such that $A \subseteq G$ always contains $X$. This fact, along with Axiom (G2), ensures the soundness of the following notion:

Definition 2. Let $(X, \mathcal{G})$ be a convex geometry. For any $A \in 2^{X}$, the convex hull of $A$ in $X$ is the smallest convex superset of $A$, that is,

$$
\operatorname{conv}_{\mathcal{G}}(A):=\bigcap\{G \in \mathcal{G}: A \subseteq G\} .
$$

Whenever the family $\mathcal{G}$ is clear from context, we shall often simplify notation, and write $\operatorname{conv}(A)$ in place of $\operatorname{conv}_{\mathcal{G}}(A)$.

The next example provides six instances of convex geometries on a three element set. It is not difficult to prove that any convex geometry on three elements is isomorphic ${ }^{3}$ to one of these. (The number of convex geometries for additional sizes of the ground set appears in the Sequence A224913 in the OEIS, which considers '(union-closed) antimatroids', that is, the structures that are complementary to convex geometries.)

Example 3. The following are convex geometries on $X=\{x, y, z\}$ :

- $\mathcal{G}_{1}=\{\varnothing,\{x\},\{x, y\}, X\}$;

- $\mathcal{G}_{2}=\{\varnothing,\{x\},\{y\},\{x, y\}, X\}$;

- $\mathcal{G}_{3}=\{\varnothing,\{x\},\{x, y\},\{x, z\}, X\}$

\footnotetext{
${ }^{3}$ Two convex geometries $\left(X, \mathcal{G}_{X}\right)$ and $\left(Y, \mathcal{G}_{Y}\right)$ are isomorphic if there is a bijection $\sigma: X \rightarrow Y$ such that, for each $A \in 2^{X}$, we have $A \in \mathcal{G}_{X}$ if and only if $\sigma(A) \in \mathcal{G}_{Y}$.
} 
- $\mathcal{G}_{4}=\{\varnothing,\{x\},\{y\},\{x, y\},\{x, z\}, X\}$;

- $\mathcal{G}_{5}=\{\varnothing,\{x\},\{y\},\{z\},\{x, y\},\{x, z\}, X\}$;

- $\mathcal{G}_{6}=2^{X}$.

There are plenty of settings where convex geometries naturally appear (under various names): see, for instance, Edelman and Jamison (1985), Goecke, Korte and Lovász (1989), Korte, Lovász, and Schrader (1991), Doignon and Falmagne (1999), and Falmagne and Doignon (2011). For nice historical overviews with reference to additional settings, see Monjardet (1985, 1990, 2008).

In the next two examples we present two important classes of convex geometries (Edelman and Jamison, 1985), namely 'ordinal' and 'affine'. In Section 4, we shall study in detail these two classes with respect to 'resolutions'.

Example 4. (Ordinal convex geometries) Let $(X, \leqslant)$ be a nonempty finite poset. Further, let $\mathcal{G}$ be the collection of all ideals of $(X, \leqslant)$, that is,

$$
\mathcal{G}:=\left\{G \in 2^{X} \mid(\forall x, y \in X)(x \leqslant y \wedge y \in G) \Longrightarrow x \in G\right\} .
$$

Then $(X, \mathcal{G})$ is a convex geometry, which is the ordinal convex geometry derived from the partial order $\leqslant$ (or, equivalently, from the poset $(X, \leqslant)$ ).

Ordinal convex geometries have a very simple characterization:

Theorem 5 (Edelman and Jamison, 1985). A convex geometry $(X, \mathcal{G})$ is ordinal if and only if $\mathcal{G}$ is closed under union. Further, if $(X, \mathcal{G})$ is a convex geometry with $\mathcal{G}$ closed under union, then there is exactly one partial order $\leqslant$ on $X$ such that $\mathcal{G}$ consists of the ideals of $\leqslant$ : in fact, $x \leqslant y$ if and only if $x \in \operatorname{conv}(\{y\})$, for all $x, y \in X$.

The partial order $\leqslant$ in Theorem 5 is said to be associated to the ordinal convex geometry $\mathcal{G}$. Notice that, for all $z, t \in X$, we have

$$
z \leqslant t \quad \Longleftrightarrow \quad z \in \operatorname{conv}(\{t\}) \text {. }
$$

Example 6. (Affine convex geometries) Let $X$ be a nonempty finite set of points in some real affine space $\mathbb{R}^{d}$. Moreover, let $\mathcal{G}$ be the collection of all sets obtained as intersections of $X$ with convex subsets of $\mathbb{R}^{d}$, that is,

$$
\mathcal{G}:=\left\{X \cap C \mid C \text { is a convex subset of } \mathbb{R}^{d}\right\}
$$

Then $(X, \mathcal{G})$ is a convex geometry, which we call affinely embedded. The family $\mathcal{G}$ is the geometry induced on the subset $X$ of $\mathbb{R}^{d}$. For any subset $A$ of $X$, we have $\operatorname{conv}_{\mathcal{G}}(A)=$ $X \cap \operatorname{conv}_{\mathbb{R}}(A)$, where conv $\mathbb{R}$ denotes the convex hull in $\mathbb{R}^{d}$. A convex geometry is affine if it is isomorphic to some affinely embedded convex geometry. 
A special feature of affine convex geometries is that they are atomistic, which means that all their one-element sets are convex (Edelman and Jamison, 1985). The problem of algorithmically characterizing affine convex geometries is nontrivial: on this topic, see Hoffmann and Merckx (2018).

Substructures of arbitrary convex geometries are defined with a procedure similar to the construction of affine convex geometries in Example 6:

Definition 7. Let $(Z, \mathcal{G})$ be a convex geometry, and $\varnothing \neq X \subseteq Z$. The convex geometry $\mathcal{H}$ induced on $X$ by $\mathcal{G}$ consists of all intersections of $X$ with elements of $\mathcal{G}{ }^{4}$ In this case, we also say that $(X, \mathcal{H})$ is a subgeometry of $(Z, \mathcal{G})$.

\section{$2.2 \quad$ Extreme Elements}

The notion of an 'extreme element' of a set is central in the theory of convex geometries (see, for instance, Edelman and Jamison, 1985).

Definition 8. Let $(X, \mathcal{G})$ be a convex geometry, and $A \in 2^{X}$. An element $a \in A$ is an extreme element of $A$ if $a \notin \operatorname{conv}(A \backslash\{a\})$. We write $\operatorname{ex}_{\mathcal{G}}(A)$ for the set of extreme elements of $A$, or simply ex $(A)$ when there is no risk of confusion. The function $\operatorname{ex}_{\mathcal{G}}: 2^{X} \rightarrow 2^{X}$ is the extreme operator on $(X, \mathcal{G})$.

Given a convex geometry $(X, \mathcal{G})$, any nonempty set $A \subseteq X$ always has at least one extreme element; in particular, $\operatorname{ex}_{\mathcal{G}}(\{x\})=\{x\}$ for any $x \in X$. Observe also that the extreme operator $\operatorname{ex}_{\mathcal{G}}: 2^{X} \rightarrow 2^{X}$ is such that $\operatorname{ex}_{\mathcal{G}}(\varnothing)=\varnothing$ and $\varnothing \neq \operatorname{ex}_{\mathcal{G}}(A) \subseteq A$ for all $A \in 2^{X} \backslash\{\varnothing\}$; that is, according to Footnote 1 or to Definition 15 below, the pair $\left(X, \operatorname{ex}_{\mathcal{G}}\right)$ is a choice space.

Example 9. We determine the extreme elements of some sets with respect to the convex geometries of Example 3. For $A=\{x, z\}$ and $B=\{y, z\}$, we have:

$$
\operatorname{ex}_{\mathcal{G}_{i}}(A)=\left\{\begin{array}{ll}
\{z\} & \text { if } 1 \leqslant i \leqslant 4 \\
A & \text { if } 5 \leqslant i \leqslant 6,
\end{array} \quad \text { and } \quad \operatorname{ex}_{\mathcal{G}_{i}}(B)= \begin{cases}\{z\} & \text { if } 1 \leqslant i \leqslant 2 \\
B & \text { if } 3 \leqslant i \leqslant 6\end{cases}\right.
$$

The next four lemmas collect several properties of the extreme operator, which will be used in later sections. Since the first three lemmas are well-known, we shall only prove the fourth.

Lemma 10. Let $(X, \mathcal{G})$ be a convex geometry. The following properties are equivalent for any $A \in 2^{X}$ and $a \in A$ :

(1) $a \in \operatorname{ex}(A)$;

(2) $\operatorname{conv}(A \backslash\{a\}) \subsetneq \operatorname{conv}(A)$;

(3) $(\exists G \in \mathcal{G})(A \backslash\{a\} \subseteq G \wedge a \notin G)$.

\footnotetext{
${ }^{4}$ The proof that $\mathcal{H}$ is a convex geometry is straightforward.
} 
Theorem 2 in Monjardet and Raderanirina (2001) yields

Lemma 11. Let $(X, \mathcal{G})$ be a convex geometry. For any $A \in 2^{X}$, we have:

(i) $\operatorname{ex}(\operatorname{conv}(A))=\operatorname{ex}(A)$;

(ii) $\operatorname{conv}(\operatorname{ex}(A))=\operatorname{conv}(A)$.

The following properties are given in Edelman and Jamison (1985):

Lemma 12. Let $(X, \mathcal{G})$ be a convex geometry. For any $G \in \mathcal{G}$ and $E \in 2^{X}$, we have:

(i) $\operatorname{ex}(G)=\{g \in G \mid G \backslash\{g\} \in \mathcal{G}\}$;

(ii) $G=\operatorname{conv}(\operatorname{ex}(G))$;

(iii) $E \subseteq \operatorname{ex}(G) \Longrightarrow G \backslash E \in \mathcal{G}$.

Finally, we prove

Lemma 13. Let $(X, \mathcal{G})$ be a convex geometry. For any $A, B \in 2^{X}$, we have:

(i) $A \subseteq B \Longrightarrow A \cap \operatorname{ex}(B) \subseteq \operatorname{ex}(A)$;

(ii) $\operatorname{ex}(A) \cap B \neq \varnothing \Longrightarrow \operatorname{ex}(A \cup B) \cap B \neq \varnothing$.

Proof. To prove (i), suppose $A \subseteq B$, and let $a \in A \cap \operatorname{ex}(B)$. By Lemma 10, there exists $G$ in $\mathcal{G}$ such that $B \backslash\{a\} \subseteq G$ and $a \notin G$. Then $A \backslash\{a\} \subseteq G$ and $a \notin G$, hence $a \in \operatorname{ex}(A)$ again by Lemma 10 .

To prove (ii), let $p \in \operatorname{ex}(A) \cap B$. Toward a contradiction, suppose $\operatorname{ex}(A \cup B) \cap B$ is empty. Then, $\operatorname{ex}(A \cup B) \subseteq A \cup B$ yields $\operatorname{ex}(A \cup B) \subseteq A$, which in turn implies $\operatorname{conv}(A \cup B) \subseteq \operatorname{conv}(A)$ by Lemma 11(ii). It follows that $B \subseteq \operatorname{conv}(A)$. On the other hand, Lemma 11(i) gives $p \in \operatorname{ex}(\operatorname{conv}(A))$. By Lemma 12(i), it follows that $\operatorname{conv}(A) \backslash\{p\}$ is a convex set. Since the latter set includes $(A \cup B) \backslash\{p\}$ but does not contain $p$, Lemma 10 entails $p \in \operatorname{ex}(A \cup B)$. We conclude that $p \in \operatorname{ex}(A \cup B) \cap B=\varnothing$, a contradiction.

Lemma 13(i) has a simple rephrasing that we will often use: If an element of a set $A$ is not extreme in $A$, then it cannot be extreme in any superset of $A$.

Remark 14. Lemma 13(ii) cannot be strengthened (as its proof might suggest) by requiring that the inclusion $\operatorname{ex}(A) \cap B \subseteq \operatorname{ex}(A \cup B) \cap B$ holds. Consider, for instance, the convex geometry $\mathcal{G}_{5}$ on $X=\{x, y, z\}$ defined in Example 3. Then, for $A:=\{x, z\}$ and $B:=X$, we have

$$
\operatorname{ex}_{\mathcal{G}_{5}}(A) \cap B=\operatorname{ex}_{\mathcal{G}_{5}}(A)=\{x, z\} \nsubseteq\{y, z\}=\operatorname{ex}_{\mathcal{G}_{5}}(B)=\operatorname{ex}_{\mathcal{G}_{5}}(A \cup B) \cap B
$$

The next definition, due to Plott (1973), introduces 'path independent choice spaces', which are important structures in mathematical economics. 
Definition 15. A function $c: 2^{X} \rightarrow 2^{X}$ such that, for any $A \in 2^{X}$,

(i) $c(A) \subseteq A$, and

(ii) $A \neq \varnothing \Longrightarrow c(A) \neq \varnothing$

is called a choice correspondence; in this case, the pair $(X, c)$ is a choice space. If, in addition, $c$ satisfies the property of path independence, i.e.,

(iii) $c(A \cup B)=c(c(A) \cup c(B))$

for any $A, B \in 2^{X}$, then $(X, c)$ is a path independent choice space.

Path independent choice spaces come up in the analysis of convex geometries because of the following striking result:

Theorem 16 (Koshevoy, 1999). If $(X, \mathcal{G})$ is a convex geometry, then $(X$, ex $)$ is a path independent choice space; in particular, for all $A, B \in 2^{X}$, we have

$$
\operatorname{ex}(A \cup B)=\operatorname{ex}(\operatorname{ex}(A) \cup \operatorname{ex}(B)) .
$$

Conversely, if $(X, c)$ is a path independent choice space, then there is a unique convex geometry $\mathcal{G}_{c}$ on $X$ whose extreme operator coincides with c, namely

$$
\mathcal{G}_{c}=\left\{G \in 2^{X} \mid\left(\forall A \in 2^{X}\right)(c(A)=c(G) \Longrightarrow A \subseteq G)\right\} .
$$

Theorem 16 allows one to establish properties of convex geometries by translating properties of path independent choice spaces. For instance, the two implications in Lemma 13 can also be derived from path independence: see, e.g., Moulin (1985) for (i), and Cantone et al. (2021) for some rephrasing of (ii). Several additional properties of the extreme operator of a convex geometry $(X, \mathcal{G})$ can also be derived as analogous features of choice correspondences. For instance, the following property (Aizerman Property) holds for any $A, B \in 2^{X}$ :

$$
\operatorname{ex}(B) \subseteq A \subseteq B \quad \Longrightarrow \quad \operatorname{ex}(A) \subseteq \operatorname{ex}(B) .
$$

In fact, Aizerman and Malishevski (1981) show that the join of the property in Lemma $13(\mathrm{i})^{5}$ and Aizerman Property is equivalent to path independence.

We close this section with two remarks about the main examples of convex geometries that will be examined in this paper.

Remark 17. Let $(X, \mathcal{G})$ be an ordinal convex geometry having $\leqslant$ as associated partial order. It is immediate to check that the extreme operator coincides with the operator of maximization w.r.t. $\leqslant$, that is, for all nonempty $A \subseteq X$,

$$
\operatorname{ex}(A)=\max (A, \leqslant):=\left\{a \in A \mid\left(\forall a^{\prime} \in A\right) a \leqslant a^{\prime} \Longrightarrow a=a^{\prime}\right\} .
$$

\footnotetext{
${ }^{5}$ This property is originally due to Chernoff (1954).
} 
In other words, the extreme elements of a set in an ordinal convex geometry are exactly the non-dominated ones. The choice space $(X, \mathrm{ex})$ that arises in view of Theorem 16 is said to be 'rationalizable' ${ }^{6}$ by the partial order $^{7} \leqslant$.

Remark 18. For a finite subset $X$ of a real affine space $\mathbb{R}^{d}$ as in Example 6 , let $\mathcal{G}$ be the convex geometry induced on $X$. Then the extreme elements of a subset $A$ of $X$ are the vertices of the polytope $\operatorname{conv}_{\mathbb{R}}(A)$. In other words, $\operatorname{ex}(A)$ is composed of all the points $a$ of $A$ with the property that some linear functional on $\mathbb{R}^{d}$ is maximized on $A$ at $a$ but at no other point of $A$.

\section{Resolutions of Convex Geometries}

Here we introduce the main notion of this paper, namely resolutions of convex geometries. This is an application of a general notion of resolution, which captures the concept of expanding a mathematical structure by substituting each of its elements by structures of the same type.

Historically, resolutions were originally defined in a topological setting by Fedorčuk (1968), and then extensively studied by Watson (1992). Resolutions have proven to be extremely useful in set-theoretic topology, providing a unified point of view for many seemingly different topological spaces.

Very recently, the notion of resolution has been adapted to the field of choice theory by Cantone et al. (2021). Upon restricting this notion to path independent choice spaces, and in view of the structural bijection provided by Theorem 16, resolutions of convex geometries naturally arise.

\subsection{Definition and Examples}

Definition 19. Let $\left(X, \mathcal{G}_{X}\right)$ be a convex geometry, which we call the base geometry, and $\left\{\left(Y_{x}, \mathcal{G}_{x}\right) \mid x \in X\right\}$ a family of convex geometries, which we call the fiber geometries. Suppose the sets $Y_{x}$ 's are pairwise disjoint, and they are also disjoint from $X$. Let

$$
Z:=\bigcup_{x \in X} Y_{x}
$$

and define the projection by

$$
\pi: Z \rightarrow X, \quad z \mapsto x \text { for all } x \in X \text { and } z \in Y_{x} .
$$

\footnotetext{
6 The rationalizability of a choice space is a well-known notion within the classical theory of revealed preferences, pioneered by the economist Samuelson (1938). Formally, a choice space $(X, c)$ is rationalizable if there is an acyclic binary relation $\lesssim$ (not necessarily a partial order) on $X$ such that, for any $A \in 2^{X}$, the equality $c(A)=\max (A, \precsim)=\left\{a \in A \mid\left(\nexists a^{\prime} \in A\right) a \prec a^{\prime}\right\}$ holds. We refer the reader to the collection of papers by Suzumura (2016) for a vast account of the topic of rationalizable choices. For some related notions of bounded rationality, which use binary relations or similar tools to explain choice behavior in different ways, see the recent survey by Giarlotta (2019) and references therein.

${ }^{7} \mathrm{~A}$ related topic is the rationalization of a choice space by means of a binary relation that is more structured than a partial order, such as one satisfying an ' $(m, n)$-Ferrers property' in the sense of Giarlotta and Watson $(2014,2018)$. On the point, it would be interesting to study (resolutions of) convex geometries that correspond to '( $m, n)$-rationalizable choices' (Cantone et al., 2016).
} 
The resolution of $\left(X, \mathcal{G}_{X}\right)$ into $\left\{\left(Y_{x}, \mathcal{G}_{x}\right) \mid x \in X\right\}$ is the pair $\left(Z, \mathcal{G}_{Z}\right)$, where $\mathcal{G}_{Z}$ consists of all subsets $A$ of $Z$ satisfying the following three requirements:

(R1) (base coherence) $\pi(A) \in \mathcal{G}_{X}$;

(R2) (fiber coherence) $A \cap Y_{x} \in \mathcal{G}_{x}$ for all $x \in \pi(A)$;

(R3) (non-extreme indiscernibility) $Y_{x} \subseteq A$ for all $x \in \pi(A) \backslash \operatorname{ex}_{\mathcal{G}_{X}}(\pi(A))$.

We shall use the suggestive notation

$$
\left(Z, \mathcal{G}_{Z}\right)=\left(X, \mathcal{G}_{X}\right) \boxminus\left(Y_{x}, \mathcal{G}_{x}\right)_{x \in X},
$$

and generically call $\left(Z, \mathcal{G}_{Z}\right)$ a resolution of convex geometries. A resolution of convex geometries is nontrivial if both the base and at least one fiber have more than one element. (See Figure 1, where each element $x$ of the base $X=\{1,2,3,4,5\}$ is resolved into a fiber $Y_{x}$, to obtain a nontrivial resolution.)

Similarly, we define the composition

$$
\left(Z, \mathcal{C}_{Z}\right)=\left(X, \mathcal{G}_{X}\right) \boxminus\left(Y_{x}, \mathcal{G}_{x}\right)_{x \in X}
$$

of the base $\left(X, \mathcal{G}_{X}\right)$ into the fibers $\left(Y_{x}, \mathcal{G}_{x}\right), x \in X$, by letting $\mathcal{C}_{Z}$ be the family of all subsets of $Z$ that satisfy (R1) and (R2) (but not necessarily (R3)). The nontriviality of a composition of convex geometries is defined similarly to resolutions.

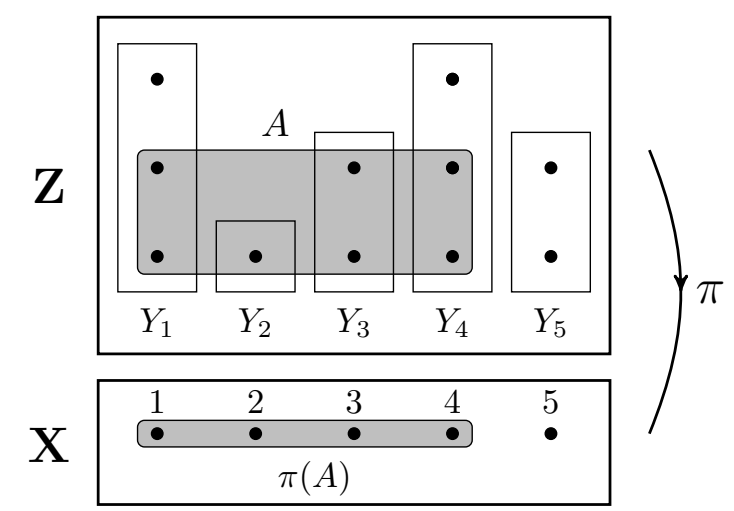

Figure 1: A nontrivial resolution $\left(Z, \mathcal{G}_{Z}\right)=\left(X, \mathcal{G}_{X}\right) \boxminus\left(Y_{x}, \mathcal{G}_{x}\right)_{x \in X}$, having $X=\{1, \ldots, 5\}$ as base set; each fiber $Y_{x}$ is above the corresponding element $x \in X$. The set $A \subseteq Z$ and its projection $\pi(A)=\{1,2,3,4\}$ are emphasized in grey.

Compositions of convex geometries are special cases of 'compound of hypergraphs' (Chein et al., 1981), and of (the equivalent notion of) 'compositions of set systems' (Möhring and Radermacher, 1984), hence also of the more abstract 'set operads' (Méndez, 2015). Contrary to what happens for resolutions, compositions do not differentiate fibers according to the status of their index (whereas resolutions do because of Requirement (R3)). This is the reason why a composition of convex geometries may fail to be a convex geometry, as the next example shows. 
Example 20. Let

$$
\begin{aligned}
X & =\{1,2\}, & \mathcal{G}_{X} & =\{\varnothing,\{1\}, X\}, \\
Y_{1} & =\left\{a_{1}, b_{1}\right\}, & \mathcal{G}_{1} & =2^{Y_{1}}, \\
Y_{2} & =\left\{a_{2}\right\}, & \mathcal{G}_{2} & =2^{Y_{2}} .
\end{aligned}
$$

Clearly, $\left(X, \mathcal{G}_{X}\right)$ and $\left(Y_{i}, \mathcal{G}_{i}\right)$, for $i=1,2$, are all convex geometries; in fact, they are even ordinal. Their (nontrivial) composition $\mathcal{C}_{Z}$, defined on $Z=\left\{a_{1}, b_{1}, a_{2}\right\}$, contains both $\left\{a_{1}, a_{2}\right\}$ and $\left\{b_{1}, a_{2}\right\}$ but not their intersection $\left\{a_{2}\right\}$, and so it fails to be a convex geometry. Instead, the resolution $\left(Z, \mathcal{G}_{Z}\right)=\left(X, \mathcal{G}_{X}\right) \boxminus\left(Y_{i}, \mathcal{G}_{i}\right)_{i=1}^{2}$ is a convex geometry (in fact, an ordinal one), because $\mathcal{G}_{Z}=\left\{\varnothing,\left\{a_{1}\right\},\left\{b_{1}\right\},\left\{a_{1}, b_{1}\right\}, Z\right\}$.

As announced in the Introduction, we have:

Theorem 21. A resolution of convex geometries is a convex geometry.

Proof. Let $\left(X, \mathcal{G}_{X}\right)$ be a convex geometry, and $\left\{\left(Y_{x}, \mathcal{G}_{x}\right) \mid x \in X\right\}$ a family of convex geometries such that the sets $Y_{x}$ 's are pairwise disjoint and disjoint from $X$. To prove that also $\left(Z, \mathcal{G}_{Z}\right)=\left(X, \mathcal{G}_{X}\right) \boxminus\left(Y_{x}, \mathcal{G}_{x}\right)_{x \in X}$ is a convex geometry, we show that $\mathcal{G}_{Z}$ satisfies Axioms (G1)-(G3) in Definition 1 by using Requirements (R1)-(R3) in Definition 19. To simplify notation, here we abbreviate $\operatorname{ex}_{\mathcal{G}_{X}}$ into ex.

(G1) The empty set is in $\mathcal{G}_{Z}$, because it trivially satisfies Requirements (R1)-(R3).

(G2) To prove that $\mathcal{G}_{Z}$ is closed under intersection, suppose $F$ and $G$ are arbitrary elements of $\mathcal{G}_{Z}$, and so they satisfy (R1)-(R3). We show that $F \cap G$ satisfies (R1)-(R3) as well.

For (R1), we prove $\pi(F \cap G) \in \mathcal{G}_{X}$. Set $E:=(\pi(F) \cap \pi(G)) \backslash \pi(F \cap G)$. Elementary computations yield $\pi(F \cap G)=(\pi(F) \backslash E) \cap(\pi(G) \backslash E)$. Since $\mathcal{G}_{X}$ is closed under intersection, it suffices to show that both $\pi(F) \backslash E$ and $\pi(G) \backslash E$ are in $\mathcal{G}_{X}$.

Claim: $E \subseteq \operatorname{ex}(\pi(F)) \cap \operatorname{ex}(\pi(G))$. Indeed, if $x \in E$, then $F \cap Y_{x} \neq \varnothing, G \cap Y_{x} \neq \varnothing$, and $\left(F \cap Y_{x}\right) \cap\left(G \cap Y_{x}\right)=\varnothing$. Then $x \in \pi(F)$ and not $Y_{x} \subseteq F$, so by (R3) $x \in \operatorname{ex}(\pi(F))$. The proof that the inclusion $E \subseteq \operatorname{ex}(\pi(G))$ holds is similar, and so the Claim is established.

Since $\pi(F), \pi(G) \in \mathcal{G}_{X}$ by (R1), now the Claim and Lemma 12(iii) allow us to conclude what we were after, namely $\pi(F) \backslash E$ and $\pi(G) \backslash E$ are in $\mathcal{G}_{X}$.

Proving (R2) is easy: we know $F \cap Y_{x} \in \mathcal{G}_{x}$ and $G \cap Y_{x} \in \mathcal{G}_{x}$, which implies $(F \cap G) \cap Y_{x}=\left(F \cap Y_{x}\right) \cap\left(G \cap Y_{x}\right)$ is in $\mathcal{G}_{x}$, as claimed.

Finally, to establish (R3), let $x \in \pi(F \cap G) \backslash \operatorname{ex}(\pi(F \cap G))$. Then $x \in \pi(F)$, and moreover $x \notin \operatorname{ex}(\pi(F))$ by Lemma 13(i) (for $A=\pi(F \cap G)$ and $B=\pi(F)$ ). Thus, since $F$ satisfies (R3), we have $Y_{x} \subseteq F$. Similarly, $Y_{x} \subseteq G$. It follows that $Y_{x} \subseteq F \cap G$, proving that $F \cap G$ satisfies (R3). 
(G3) To show that $\mathcal{G}_{Z}$ is upgradable, let $F \in \mathcal{G}_{Z} \backslash\{Z\}$. If $F$ hits some fiber $Y_{x}$ without including all of it, then $F \cap Y_{x}$ belongs to $\mathcal{G}_{x}$ by (R2), hence, by the upgradability of the convex geometry $\mathcal{G}_{x}$, there is some $y$ in $Y_{x} \backslash F$ such that $\left(F \cap Y_{x}\right) \cup\{y\} \in \mathcal{G}_{x}$. It follows that $F \cup\{y\}$ is in $\mathcal{G}_{Z}$. On the other hand, if $F$ is a union of fibers, then, since $F \neq Z$ and $\pi(F) \in \mathcal{G}_{X}$, there is $x$ in $X \backslash \pi(F)$ such that $\pi(F) \cup\{x\} \in \mathcal{G}_{X}$. There exists $y$ in $Y_{x}$ such that $\{y\} \in \mathcal{G}_{x}$. Then $F \cup\{y\} \in \mathcal{G}_{Z}$ (because $x \in \operatorname{ex}(\pi(F \cup\{y\}))$.

This completes the proof.

Remark 22. Let $\left(Z, \mathcal{G}_{Z}\right)=\left(X, \mathcal{G}_{X}\right) \boxminus\left(Y_{x}, \mathcal{G}_{x}\right)_{x \in X}$ be a resolution of convex geometries. For any $A \in 2^{X}$, we have

$$
\pi^{-1}(A) \in \mathcal{G}_{Z} \quad \Longleftrightarrow \quad A \in \mathcal{G}_{X}
$$

hence, in particular, for any $x \in X$,

$$
\mathcal{G}_{x} \subseteq \mathcal{G}_{Z} \quad \Longleftrightarrow \quad Y_{x} \in \mathcal{G}_{Z} \quad \Longleftrightarrow \quad\{x\} \in \mathcal{G}_{X} .
$$

(To prove that $Y_{x} \in \mathcal{G}_{Z}$ implies $\mathcal{G}_{x} \subseteq \mathcal{G}_{Z}$, observe that Requirement (R3) is vacuously satisfied, because the unique element of $\{x\}$ is extreme.) Also, the resolution is atomistic if and only if the base and all fibers are atomistic. Moreover, for any $x \in X$, the equivalence

$$
Z \backslash Y_{x} \in \mathcal{G}_{Z} \quad \Longleftrightarrow \quad x \in \operatorname{ex}_{\mathcal{G}_{X}}(X)
$$

holds. Any fiber $\left(Y_{x}, \mathcal{G}_{x}\right)$ is a subgeometry of $\left(Z, \mathcal{G}_{Z}\right)$. Furthermore, if $S \subseteq Z$ is a transversal (i.e., $S$ intersects each fiber in exactly one element), then the convex geometry induced by $\left(Z, \mathcal{G}_{Z}\right)$ on $S$ is isomorphic to $\left(X, \mathcal{G}_{X}\right)$.

We shall show in Section 4 that the two main classes of convex geometries examined in this paper, namely ordinal (Example 4) and affine (Example 6), behave in a radically different way with respect to resolutions: in fact, ordinality is preserved whereas affineness is not. Specifically, the resolution of ordinal convex geometries is again ordinal, and this procedure encapsulates a well-known construction on posets (Theorem 54). On the other hand, we shall provide an elementary example of a resolution of affine convex geometries that fails to be affine (Example 42).

We conclude these preliminaries with a technical result, which describes the extreme operator and the convex hull operator in a resolution.

Lemma 23. Let $\left(Z, \mathcal{G}_{Z}\right)=\left(X, \mathcal{G}_{X}\right) \boxminus\left(Y_{x}, \mathcal{G}_{x}\right)_{x \in X}$ be a resolution of convex geometries. For any $A \subseteq Z$, we have

$$
\operatorname{conv}_{\mathcal{G}_{Z}}(A)=\bigcup_{x \in \operatorname{ex}_{\mathcal{G}_{X}}(\pi(A))} \operatorname{conv}_{\mathcal{G}_{x}}\left(A \cap Y_{x}\right) \cup \bigcup_{x \in \operatorname{conv}_{\mathcal{G}_{X}}(\pi(A)) \backslash \operatorname{ex}_{\mathcal{G}_{X}}(\pi(A))} Y_{x}
$$

and

$$
\operatorname{ex}_{\mathcal{G}_{Z}}(A)=\bigcup_{x \in \operatorname{ex}_{\mathcal{G}_{X}}(\pi(A))} \operatorname{ex}_{\mathcal{G}_{x}}\left(A \cap Y_{x}\right)
$$


Proof. Let $A \in 2^{Z}$. We prove (3). To start, we show that the right-hand side of (3) belongs to $\mathcal{G}_{Z}$. Indeed, it satisfies (R1), because its projection is equal to $\operatorname{conv}_{\mathcal{G}_{X}}(\pi(A))$, and so it is in $\mathcal{G}_{X}$. Moreover, it satisfies (R2) and (R3), too: use the special case $\operatorname{ex}_{\mathcal{G}_{X}}\left(\operatorname{conv}_{\mathcal{G}_{X}}(\pi(A))\right)=\operatorname{ex}_{\mathcal{G}_{X}}(\pi(A))$ of Lemma 11(i). This proves that the right-hand side of (3) is convex. Therefore, to complete the proof of (3), it suffices to show that any convex set $G$ in $\mathcal{G}_{Z}$ that includes $A$ also includes the right-hand side.

To that end, let $G \in \mathcal{G}_{Z}$ be such that $A \subseteq G$. Take any $x \in \operatorname{ex}_{\mathcal{G}_{X}}(\pi(A))$. Since the convex set $G$ includes $A \cap Y_{x}$, it follows that $G \cap Y_{x}$ is a convex set in $Y_{x}$ including $A \cap Y_{x}$, and so $\operatorname{conv}_{\mathcal{G}_{x}}\left(A \cap Y_{x}\right) \subseteq G \cap Y_{x} \subseteq G$. On the other hand, let $x \in \operatorname{conv}_{\mathcal{G}_{X}}(\pi(A)) \backslash \operatorname{ex}_{\mathcal{G}_{X}}(\pi(A))$. Observe that Requirement (R1) yields $\pi(A) \subseteq \pi(G) \in \mathcal{G}_{X}$, hence $\operatorname{conv}_{\mathcal{G}_{X}}(\pi(A)) \subseteq \pi(G)$. Since $\operatorname{ex}_{\mathcal{G}_{X}}(\pi(G)) \cap \operatorname{conv}_{\mathcal{G}_{X}}(\pi(A)) \subseteq \operatorname{ex}_{\mathcal{G}_{X}}(\pi(A))$ by Lemma 13(i), it follows that $x \in$ $\pi(G) \backslash \operatorname{ex}_{\mathcal{G}_{X}}(\pi(G))$, and so $Y_{x} \subseteq G$ by Requirement (R3). This proves that (3) holds.

Next, we prove (4). For the forward inclusion, let $z \in \operatorname{ex}_{\mathcal{G}_{Z}}(A)$. Set $x:=\pi(z)$. To prove the claim, we show that (i) $x \in \operatorname{ex}_{\mathcal{G}_{X}}(\pi(A))$, and (ii) $z \in \operatorname{ex}_{\mathcal{G}_{x}}\left(A \cap Y_{x}\right)$. By Lemma 10(3), there exists $G \in \mathcal{G}_{Z}$ such that $A \backslash\{z\} \subseteq G$ and $z \notin G$. Now, if $G \cap Y_{x} \neq \varnothing$, then $Y_{x} \nsubseteq G$, and so $x \in \operatorname{ex}_{\mathcal{G}_{X}}(\pi(G))$ by Requirement (R3). It follows that $x \in \operatorname{ex}_{\mathcal{G}_{X}}(\pi(A))$ by Lemma 13(i). On the other hand, suppose $G \cap Y_{x}=\varnothing$. Then the set $\pi(G)$ is convex in $\mathcal{G}_{X}$ and includes $\pi(A) \backslash\{x\}$ but not $x$, which again implies $x \in \operatorname{ex}_{\mathcal{G}_{X}}(\pi(A))$. This proves (i). For (ii), observe that $G \cap Y_{x}$ is convex in $Y_{x}$, includes $\left(A \cap Y_{x}\right) \backslash\{z\}$ but does not contain $z$; therefore, $z \in \operatorname{ex}_{x}\left(A \cap Y_{x}\right)$. This completes the proof of the forward inclusion in (4).

For the reverse inclusion, let $x \in \operatorname{ex}_{\mathcal{G}_{X}}(\pi(A))$ and $z \in \operatorname{ex}_{\mathcal{G}_{x}}\left(A \cap Y_{x}\right)$. It suffices to show $z \notin \operatorname{conv}_{\mathcal{G}_{Z}}(A \backslash\{z\})$, which we do by applying (3) to $A \backslash\{z\}$ in two exhaustive cases. If $(A \backslash\{z\}) \cap Y_{x}=\varnothing$, then $x \notin \operatorname{conv}_{\mathcal{G}_{x}}\left(\pi(A \backslash\{z\})\right.$ because $x \in \operatorname{ex}_{\mathcal{G}_{x}}(\pi(A))$, and so $z \notin$ $\operatorname{conv}_{\mathcal{G}_{Z}}(A \backslash\{z\})$. If $(A \backslash\{z\}) \cap Y_{x} \neq \varnothing$, then $\pi(A \backslash\{z\})=\pi(A)$, so $x \in \operatorname{ex}_{\mathcal{G}_{x}}(\pi(A \backslash\{z\}))$. Together with $z \notin \operatorname{conv}_{\mathcal{G}_{x}}\left((A \backslash\{z\}) \cap Y_{x}\right)$, this gives again $z \notin \operatorname{conv}_{\mathcal{G}_{Z}}(A \backslash\{z\})$.

\subsection{Primitivity vs Resolvability. Shrinkable Sets}

Using resolutions, convex geometries can be partitioned in two classes:

Definition 24. A convex geometry is primitive (or irresolvable) if it cannot be obtained as a nontrivial resolution; otherwise, it is resolvable.

How can we directly recognize whether a given convex geometry is primitive or resolvable? Definition 19 implies that the convex geometry resulting from a resolution is partitioned into fibers. Then a naive algorithm to test primitiveness should check one by one all nontrivial partitions of the convex geometry, derive the corresponding base and fibers, and stop when their resolution is the initial convex geometry. Obviously, this algorithm is highly ineffective. A better algorithm can be derived from Theorem 26 below. To state it, we need the following notion of 'shrinkability':

Definition 25. Let $(Z, \mathcal{G})$ be a convex geometry. A subset $S$ of $Z$ is shrinkable (in $Z$ ) if it is a nontrivial fiber in some nontrivial resolution producing $(Z, \mathcal{G})$. 
Observe that if $S$ is shrinkable in $X$, then $1<|S|<|X|$. Clearly, a convex geometry is primitive if and only if it has no shrinkable set. The following characterization of shrinkability yields a more effective test for the primitiveness of a convex geometry:

Theorem 26. Let $\left(Z, \mathcal{G}_{Z}\right)$ be a convex geometry. The following statements are equivalent for a subset $S$ of $Z$ such that $1<|S|<|Z|$ :

(i) $S$ is shrinkable;

(ii) S satisfies the following two properties:

(S1) $\left(\forall G \in \mathcal{G}_{Z}\right) \quad\left(G \cap S \neq S \Longrightarrow G \backslash S \in \mathcal{G}_{Z}\right)$;

(S2) $\left(\forall G, H \in \mathcal{G}_{Z}\right) \quad\left(G \cap S \neq \varnothing \wedge G \backslash S \in \mathcal{G}_{Z}\right) \Longrightarrow(G \backslash S) \cup(H \cap S) \in \mathcal{G}_{Z}$.

Proof. (i) $\Longrightarrow$ (ii). Suppose $S$ is shrinkable. Thus, $\left(Z, \mathcal{G}_{Z}\right)$ can be written as

$$
\left(Z, \mathcal{G}_{Z}\right)=\left(X, \mathcal{G}_{X}\right) \boxminus\left(Y_{x}, \mathcal{G}_{x}\right)_{x \in X},
$$

where $S=Y_{s}$ for some $s \in X$. Let $G, H \in \mathcal{G}_{Z}$, hence $\pi(G), \pi(H) \in \mathcal{G}_{X}$ by (R1). Below we show that Properties (S1) and (S2) hold for $S=Y_{s}$.

For (S1), suppose $G \in \mathcal{G}_{Z}$ is such that $\varnothing \neq G \cap Y_{s} \neq Y_{s}$ (if $G \cap Y_{s}=\varnothing$, then the result holds trivially). Then $s \in \operatorname{ex}_{\mathcal{G}_{X}}(\pi(G))$ by (R3), hence Lemma 12(i) yields $\pi\left(G \backslash Y_{s}\right)=\pi(G) \backslash\{s\} \in \mathcal{G}_{X}$, which proves that (R1) holds for $G \backslash Y_{s}$. Furthermore, $G \backslash Y_{s}$ satisfies Requirement (R2), because so does $G$. Thus, to prove that $G \backslash Y_{s} \in \mathcal{G}_{Z}$, it remains to show that also Requirement (R3) holds for $G \backslash Y_{s}$. To that end, observe that Lemma 13(i) yields $\pi\left(G \backslash Y_{s}\right) \cap \operatorname{ex}_{\mathcal{G}_{X}}(\pi(G)) \subseteq \operatorname{ex}_{\mathcal{G}_{X}}\left(\pi\left(G \backslash Y_{s}\right)\right)$. It follows that any non-extreme element of $\pi\left(G \backslash Y_{s}\right)$ is also non-extreme in $\pi(G)$. Hence $G \backslash Y$ satisfies (R3) because so does $G$.

For (S2), suppose $G \in \mathcal{G}_{Z}$ satisfies $G \cap Y_{s} \neq \varnothing$ and $G \backslash Y_{s} \in \mathcal{G}_{Z}$. If $H \cap Y_{s}=\varnothing$, then (S2) holds. Thus, let $H \cap Y_{s} \neq \varnothing$. Below we show that $\left(G \backslash Y_{s}\right) \cup\left(H \cap Y_{s}\right)$ is in $\mathcal{G}_{Z}$ by proving that Requirements (R1)-(R3) hold for it. Requirement (R1) readily follows from $\pi\left(\left(G \backslash Y_{s}\right) \cup\left(H \cap Y_{s}\right)\right)=\pi(G) \in \mathcal{G}_{X}$. Requirement (R2) holds, because both $G$ and $H$ satisfy it. Finally, for (R3), observe that $s$ is an extreme element for $\left.\pi\left(G \backslash Y_{s}\right) \cup\left(H \cap Y_{s}\right)\right)=\pi(G)$, because $\pi(G), \pi(G) \backslash\{s\} \in \mathcal{G}_{X}$. Thus, $\left(G \backslash Y_{s}\right) \cup\left(H \cap Y_{s}\right)$ satisfies Requirement (R3), because so does $G$.

(ii) $\Longrightarrow\left(\right.$ i). Suppose $S$ is a subset of the convex geometry $\left(Z, \mathcal{G}_{Z}\right)$ such that $1<|S|<$ $|Z|$, and Properties (S1) and (S2) hold for all $G, H \in \mathcal{G}_{Z}$. To show that $S$ is shrinkable, we shall express $\left(Z, \mathcal{G}_{Z}\right)$ as a nontrivial resolution having $S$ as its unique nontrivial fiber.

Select $s \notin Z$. Let $\sigma: Z \backslash S \rightarrow W$ be a bijection from $Z \backslash S$ onto a set $W$ that is disjoint from $Z \cup\{s\}$, and denote $x_{z}:=\sigma(z)$ for all $z \in Z \backslash S$. As base set, take $X:=W \cup\{s\}$. Notice that $X \cap Z=\varnothing$ by construction, and $|X| \geqslant 2$ because $S \neq Z$ by hypothesis. As fiber sets, take $Y_{s}:=S$, and $Y_{x_{z}}:=\{z\}$ for all $x_{z} \in W=X \backslash\{s\}$. Therefore, $Z$ is equal to $\bigcup_{x \in X} Y_{x}$, where $|X| \geqslant 2$ and $\left|Y_{s}\right| \geqslant 2$. As usual, let $\pi: Z \rightarrow X$ be the (projection) map defined by $\pi(z):=x_{z}$ if $z \in Z \backslash S$, and $\pi(z):=s$ if $z \in S$. Next, we endow both the base set $X$ and the fiber sets $Y_{x}$ 's with convex geometries induced by $\mathcal{G}_{Z}$. 
Concerning the base, let $\mathcal{G}_{X}$ be the family of subsets of $X$ defined by

$$
\mathcal{G}_{X}:=\left\{\pi(G) \mid G \in \mathcal{G}_{Z}\right\} .
$$

We now check that $\left(X, \mathcal{G}_{X}\right)$ is a convex geometry. To begin with, observe that $\pi(\varnothing)=$ $\varnothing \in \mathcal{G}_{X}$, that is, Axiom (G1) holds.

To prove Axiom (G2) (closure under intersection), we let $\pi(G), \pi(H) \in \mathcal{G}_{X}$, where $G, H \in \mathcal{G}_{Z}$, and show that $\pi(G) \cap \pi(H) \in \mathcal{G}_{X}$. We know that $\pi(G \cap H) \subseteq \pi(G) \cap \pi(H)$. If equality holds, then we are immediately done, since $G \cap H \in \mathcal{G}_{Z}$. Otherwise, we must have $\varnothing \neq G \cap S \neq S, \varnothing \neq H \cap S \neq S,(G \cap H) \cap S=\varnothing$, and $\pi(G) \cap \pi(H)=\pi(G \cap H) \cup\{s\}$. Now apply Property (S1) to $G$ and next Property (S2) to $G$ and $H$, to derive that the set $K:=(G \backslash S) \cup(H \cap S)$ is an element of $\mathcal{G}_{Z}$. Since $K \cap H \in \mathcal{G}_{Z}$, and $\pi(G) \cap \pi(H)=$ $\pi(G \cap H) \cup\{s\}=\pi(K \cap H)$, we conclude that $\pi(G) \cap \pi(H)$ is an element of $\mathcal{G}_{X}$ also in this case, as claimed. This shows that $\mathcal{G}_{X}$ satisfies Axiom (G2).

To prove Axiom (G3), let $\pi(G) \neq X$, with $G \in \mathcal{G}_{Z}$. By Axiom (G3) for $\left(Z, \mathcal{G}_{Z}\right)$, there is $z_{1} \in Z \backslash G$ such that $G_{1}:=G \cup\left\{z_{1}\right\} \in \mathcal{G}_{Z}$. If $G \cap S$ is either empty or equal to $S$, there holds $\pi\left(z_{1}\right) \notin \pi(G)$, and the set $\pi(G) \cup\left\{\pi\left(z_{1}\right)\right\}=\pi\left(G_{1}\right) \in \mathcal{G}_{X}$ is what we were looking for. On the other hand, suppose $\varnothing \neq G \cap S \neq S$. If $z_{1} \notin S$, then $\pi\left(G_{1}\right)=\pi(G) \cup\left\{x_{z_{1}}\right\}$, and we are done. Otherwise, there is $z_{2} \in Z \backslash G_{1}$ such that $G_{2}:=G_{1} \cup\left\{z_{2}\right\} \in \mathcal{G}_{Z}$. Again, if $z_{2} \notin S$, the claim holds. Otherwise, we iterate. Since $\pi(G) \neq X$ and $X$ is finite, we shall eventually get $G_{n}:=G_{n-1} \cup\left\{z_{n}\right\} \in \mathcal{G}_{Z}$ such that $\pi\left(G_{i}\right)=\pi(G)$ for all $i=1, \ldots, n-1$, and $z_{n} \notin S$. Then $\pi\left(G_{n}\right)=\pi(G) \cup\left\{x_{z_{n}}\right\}$ proves that $\mathcal{G}_{X}$ satisfies (G3).

To complete the definition of the resolution, we provide each fiber $Y_{x}$ with the convex geometry $\mathcal{G}_{x}$ induced by $\mathcal{G}_{Z}$, that is,

$$
\mathcal{G}_{x}:= \begin{cases}\{\varnothing,\{x\}\} & \text { if } x \in W \\ \left\{G \cap S \mid G \in \mathcal{G}_{Z}\right\} & \text { if } x=s\end{cases}
$$

Below we show that $\left(Z, \mathcal{G}_{Z}\right)=\left(X, \mathcal{G}_{X}\right) \boxminus\left(Y_{x}, \mathcal{G}_{x}\right)_{x \in X}$, thus completing the proof.

To start, we prove that any convex set $G$ from $\mathcal{G}_{Z}$ is also convex in the resolution $\left(X, \mathcal{G}_{X}\right) \boxminus\left(Y_{x}, \mathcal{G}_{x}\right)_{x \in X}$, that is, $G$ satisfies Requirements (R1)-(R3). Clearly $\pi(G) \in \mathcal{G}_{X}$ by the very definition of $\mathcal{G}_{X}$, thus (R1) holds for $G$. Furthermore, the intersection of $G$ with an arbitrary fiber $Y_{x}$ is convex in $Y_{x}$ by definition of $\mathcal{G}_{x}$, so $G$ also satisfies (R2). Finally, if $G \cap S=\varnothing$ or $G \cap S=S$, then (R3) holds trivially for $G$. On the other hand, if $\varnothing \neq G \cap S \neq S$, then we can apply Property (S1) to $S=Y_{s}$ to get $G \backslash S \in \mathcal{G}_{Z}$. Since $\{s\}=\pi(G) \backslash \pi(G \backslash S)$, we obtain that $s$ is an extreme element in the convex set $\pi(G) \in \mathcal{G}_{X}$. We conclude that $G$ satisfies (R3) also in this case, and so $G$ is convex in the resolution $\left(X, \mathcal{G}_{X}\right) \boxminus\left(Y_{x}, \mathcal{G}_{x}\right)_{x \in X}$

For the reverse inclusion, we show that if $K$ is a nonempty convex set in $\left(X, \mathcal{G}_{X}\right) \boxminus$ $\left(Y_{x}, \mathcal{G}_{x}\right)_{x \in X}$, then $K \in \mathcal{G}_{Z}$. Since $\pi(K) \in \mathcal{G}_{X}$ by Requirement (R1) of a resolution, there is $G \in \mathcal{G}_{Z}$ such that $\pi(K)=\pi(G)$. Clearly, $K \cap Y_{x}=G \cap Y_{x}$ for all $x \in W$. If also $K \cap S=G \cap S$ holds, then we have $K=G$, and we are immediately done. Since the latter fact trivially holds if $K$ and $S$ are disjoint, we may assume that $K \cap S$ is different from both $\varnothing$ and $G \cap S$ (so $G \cap S$ is nonempty, too). Next we appeal to Requirement (R2), 
and get $K \cap S \in \mathcal{G}_{s}$, hence, by the very definition of $\mathcal{G}_{s}$, there is $H \in \mathcal{G}_{Z}$ such that $K \cap S=H \cap S \neq \varnothing$. It follows that $K=(G \backslash S) \cup(H \cap S)$. To conclude our proof, we deal separately with the following two exhaustive cases.

(1) In case $S \nsubseteq G$, we have $G \cap S \neq S$, hence Property (S1) yields $G \backslash S \in \mathcal{G}_{Z}$. Since $G \cap S \neq \varnothing$, Property (S2) entails $K=(G \backslash S) \cup(H \cap S) \in \mathcal{G}_{Z}$, as required.

(2) In case $S \subseteq G$, we have $S \nsubseteq K$, because $K \cap S$ differs from $G \cap S$. Then, by Requirement (R3), $s$ is extreme in $\pi(K)$. The latter set is convex in $X$, and moreover it is equal to $\pi(G)$. We derive that $\pi(G) \backslash\{s\}$ is also convex in $\mathcal{G}_{X}$, and next that $G \backslash S$ belongs to $\mathcal{G}_{Z}$ (because $\pi(G) \backslash\{s\}$ must be the image by $\pi$ of a convex set from $\mathcal{G}_{Z}$, which must be $G \backslash S)$. By (S2) we conclude $K=(G \backslash S) \cup(H \cap S) \in \mathcal{G}_{Z}$, as required.

Remark 27. If in (S2) we replace $G \cap S \neq \varnothing$ with $|G \cap S|=1$, then we get an equivalent condition. Indeed, assume the modified (S2) is true, and let us prove (S2) in case $|G \cap S|>$ 1. Since both $G \backslash S$ and $G$ are convex by assumption, there exists some $s \in G \cap S$ such that $G^{\prime}:=(G \backslash S) \cup\{s\} \in \mathcal{G}_{Z}$ (this follows from the axioms of a convex geometry). By assumption the modified (S2) holds for $G^{\prime}$. As we have $(G \backslash S) \cup(H \cap S)=\left(G^{\prime} \backslash S\right) \cup(H \cap S)$, it follows that (S2) holds for $G$. This completes the proof of the equivalence of the two conditions. Finally, observe that in the modified (S2), the element $s$ forming $G \cap S$ is an extreme element of $G$, because $G$ and $G \backslash\{s\}$ are convex.

Properties (S1) and (S2) are logically independent, even on some (small) affine convex geometry $(Z, \mathcal{G})$. The next example shows such independence, at the same time exhibiting a resolvable, affine convex geometry.

Example 28. Let $Z$ consist of four points $a, b, c, d$ in the real affine plane, where $c$ belongs to the segment having $b$ and $d$ as extreme points, and $a$ is outside the line passing through the other three points (see Figure 2). Consider the affine convex geometry $\mathcal{G}_{Z}$ induced on $Z$.

To show that (S1) and (S2) are mutually independent properties, let $S^{\prime}=\{a, b\}$ and $S^{\prime \prime}=\{a, c\}$. Then, $S^{\prime}$ satisfies (S1) (because all subsets of $Z \backslash S^{\prime}$ are convex) but not (S2) (take $G=\{a, d\}$ and $H=\{b\}$ ). On the other hand, $S^{\prime \prime}$ does not satisfy (S1) (take $G=\{b, c, d\}$ ) but satisfies (S2) (because there are only two sets in $Z$ that are not convex, namely $\{b, d\}$ and $\{a, b, d\})$.

Observe also that $S=\{b, c, d\}$ is a shrinkable set in $\left(Z, \mathcal{G}_{Z}\right)$, which is therefore a resolvable convex geometry. In fact, it is easy to check that $\left(Z, \mathcal{G}_{Z}\right)$ is the resolution with base $\left(\{1,2\}, 2^{\{1,2\}}\right)$ and fibers $\left(Y_{1}, \mathcal{G}_{1}\right)$ and $\left(Y_{2}, \mathcal{G}_{2}\right)$, where

$$
\begin{array}{ll}
Y_{1}=\{a\}, & \mathcal{G}_{1}=2^{Y_{1}}, \\
Y_{2}=\{b, c, d\}, & \mathcal{G}_{2}=2^{Y_{2}} \backslash\{b, d\} .
\end{array}
$$

Here, the base, the fibers, and the resolution are affine convex geometries.

For compositions (or compounds) of hypergraphs, 'committees' play a role akin to our shrinkable sets. According to Chein et al. (1981), a committee in a hypergraph $(X, \mathcal{G})$ (where by definition $\mathcal{G} \subseteq 2^{X}$ ) is any subset $S$ of $X$ satisfying a property similar to (S2) in Theorem 26, where the antecedent is $G \cap S \neq \varnothing$ and $H \cap S \neq \varnothing$. 


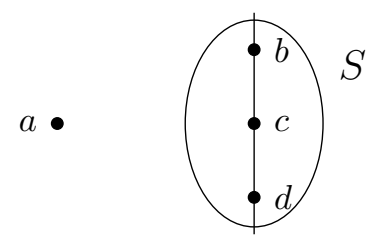

Figure 2: Four points in the real affine plane; the induced convex geometry on $\{a, b, c, d\}$ admits $S$ as a shrinkable set (see Example 28).

Cantone et al. (2021) characterize shrinkable sets in the more general setting of choice spaces. In view of Koshevoy's result (Theorem 16) and a simplification due to the path independence of the associated choice space, this directly yields an alternative test for the primitiveness of a convex geometry which employs the extreme operator.

Theorem 29. Let $(Z, \mathcal{G})$ be a convex geometry. The following statements are equivalent for a subset $S$ of $Z$ such that $1<|S|<|Z|$ :

(i) $S$ is shrinkable;

(ii) $S$ satisfies the following properties for any $A \in 2^{Z}$ :

$(\mathrm{T} 1) \operatorname{ex}_{\mathcal{G}}(A) \cap S \neq \varnothing \Longrightarrow \operatorname{ex}_{\mathcal{G}}(A \cap S) \subseteq \operatorname{ex}_{\mathcal{G}}(A) ;$

(T2) $A \cap S \neq \varnothing \Longrightarrow \operatorname{ex}_{\mathcal{G}}(A) \backslash S \subseteq \operatorname{ex}_{\mathcal{G}}(A \cup S)$;

(T3) $\left(A \cap S \neq \varnothing \wedge \operatorname{ex}_{\mathcal{G}}(A \cup S) \cap S \neq \varnothing\right) \Longrightarrow \operatorname{ex}_{\mathcal{G}}(A) \cap S \neq \varnothing$.

Moreover, when $(Z, \mathcal{G})$ is atomistic, (T1) implies (T3), and so Properties (T1) and (T2) characterize the shrinkability of $S$.

We now establish the independence among the three Properties (T1)-(T3).

Example 30. As in Example 28, consider the affine convex geometry induced on four points $a, b, c$ and $d$ in the real affine plane, with $c$ between $b$ and $d$ and moreover $a$ outside the line through $b, c$ and $d$. If we let $S=\{a, b, c\}$, then $S$ does not satisfy (T1) (for $A=\{a, b, c\}$ ) but it satisfies (T2) and (T3). If we now let $S=\{a, d\}$, then $S$ satisfies (T1) and thus also (T3), but not (T2) (for $A=\{a, b, c\}$ ). Thus even in affine convex geometries, (T1) and (T2) are each one independent of the other two properties.

To show that (T3) is independent of (T1) and (T2), consider the ordinal convex geometry derived from the partial order $\leqslant$ on $Z=\{a, b, c\}$ with $a<b, a<c$ and no other strict comparison. Then the subset $S=\{a, b\}$ satisfies (T1) and (T2), but not (T3) (take $A=\{a, c\})$.

\subsection{Extreme Resolutions and Extremely Shrinkable Sets}

Here we study resolutions whose nontrivial fibers are all indexed by extreme elements of the base. 
Definition 31. A resolution

$$
\left(Z, \mathcal{G}_{Z}\right)=\left(X, \mathcal{G}_{X}\right) \boxminus\left(Y_{x}, \mathcal{G}_{x}\right)_{x \in X}
$$

is extreme when for each $x \in X$, if $x \notin \operatorname{ex}_{\mathcal{G}_{X}}(X)$ then $\left|Y_{x}\right|=1$.

As we shall see in Theorem 43, any resolution of convex geometries that happens to be affine is also extreme. The next result uses extremeness to further clarify the link between compositions and resolutions.

Theorem 32. Let $\left(Z, \mathcal{G}_{Z}\right)=\left(X, \mathcal{G}_{X}\right) \boxminus\left(Y_{x}, \mathcal{G}_{x}\right)_{x \in X}$ be a resolution of convex geometries, and $\left(Z, \mathcal{C}_{Z}\right)=\left(X, \mathcal{G}_{X}\right) \boxminus\left(Y_{x}, \mathcal{G}_{x}\right)_{x \in X}$ the composition with the same base and fibers. Then $\mathcal{G}_{Z} \subseteq \mathcal{C}_{Z}$, and $\mathcal{G}_{Z}=\mathcal{C}_{Z}$ holds if and only if the resolution is extreme.

Proof. Definition 19 readily yields $\mathcal{G}_{Z} \subseteq \mathcal{C}_{Z}$. Next we show that if the resolution is extreme, then $\mathcal{C}_{Z} \subseteq \mathcal{G}_{Z}$. Indeed, in an extreme resolution, any subset $A$ of $Z$ satisfies Requirement (R3), because an element $x$ that is non-extreme in $\pi(A)$ is also non-extreme in $X$, hence $Y_{x}$ has only one element and is contained in $A$.

Conversely, we show that if the resolution is not extreme, then $\mathcal{C}_{Z} \subseteq \mathcal{G}_{Z}$ does not hold. By assumption, there exists some $x$ in $X \backslash \operatorname{ex}_{\mathcal{G}_{x}}(X)$ such that the fiber $Y_{x}$ contains at least two elements. By the definition of a convex geometry, $\mathcal{G}_{Y_{x}}$ contains a nonempty convex set $G$ distinct from $Y_{x}$. Now it is easy to check that $\left(Z \backslash Y_{x}\right) \cup G$ belongs to $\mathcal{C}_{Z}$ but not to $\mathcal{G}_{Z}$.

Remark 33. Theorem 32 has a direct extension to a large family of set systems, as we now explain. A set system is a pair $(X, \mathcal{F})$, where $X$ is a nonempty set, and $\mathcal{F}$ is a nonempty collection of subsets of $X$. A set system $(X, \mathcal{F})$ is simple if $\bigcup \mathcal{F}=X$; in particular, $(X, \mathcal{F})$ is plain if it is simple and moreover $\mathcal{F} \neq\{\varnothing, X\}$ whenever $|X|>1$.

Compositions of set systems are defined exactly as compositions of convex geometries (using only Conditions (R1) and (R2) as in Definition 19). To define resolutions of set systems, we only need a notion of extreme element in set systems, and then again copy from Definition 19. Lemma 10 suggests the following definition: given a set system $(X, \mathcal{F})$, an element $x$ in a subset $A$ of $X$ is extreme in $A$ when there exists some $F \in \mathcal{F}$ such that $A \backslash\{x\} \subseteq F$ and $x \notin F$ (compare with, for instance, Ando, 2006).

Now consider a composition $\left(Z, \mathcal{F}_{Z}\right)=\left(X, \mathcal{F}_{X}\right) \boxminus\left(Y_{x}, \mathcal{F}_{x}\right)_{x \in X}$ and at the same time a resolution $\left(Z, \mathcal{C}_{Z}\right)=\left(X, \mathcal{F}_{X}\right) \boxminus\left(Y_{x}, \mathcal{F}_{x}\right)_{x \in X}$ of set systems (with the same base and fibers). As for convex geometries (Theorem 32), the resolution $\mathcal{F}_{Z}$ is a subcollection of the composition $\mathcal{C}_{Z}$. For plain set systems, the arguments in the proof of Theorem 32 show that the equality $\mathcal{F}_{Z}=\mathcal{C}_{Z}$ occurs exactly when the following property is satisfied: for any nontrivial fiber $Y_{x}$ and convex set $F$ in $\mathcal{F}_{X}$ containing $x$, there holds $x \in \operatorname{ex}_{\mathcal{F}_{X}}(F)$. (Observe that if $X \in \mathcal{F}_{X}$, it suffices to require this property to hold for $F=X$, because an element $x$ that is extreme in $X$ is also extreme in any subset of $X$ containing $x$.)

To recognize which convex geometries can be written as a nontrivial extreme resolution, we introduce and characterize a variant of shrinkability. 
Definition 34. Let $\left(Z, \mathcal{G}_{Z}\right)$ be a convex geometry. A subset $S$ of $Z$ with at least two elements is extremely shrinkable (in $Z$ ) if it is a fiber in some nontrivial extreme resolution producing $\left(Z, \mathcal{G}_{Z}\right)$. Whenever $Z$ contains such a set, we say that $\left(Z, \mathcal{G}_{Z}\right)$ is extremely resolvable.

Theorem 35. Let $\left(Z, \mathcal{G}_{Z}\right)$ be a convex geometry. The following statements are equivalent for a shrinkable set $S \subseteq Z$ :

(i) $S$ is extremely shrinkable;

(ii) for any resolution $\left(X, \mathcal{G}_{X}\right) \boxminus\left(Y_{x}, \mathcal{G}_{x}\right)_{x \in X}$ equal to $\left(Z, \mathcal{G}_{Z}\right)$, if $S$ coincides with a fiber $Y_{x}$, then $x \in \operatorname{ex}_{\mathcal{G}_{X}}(X)$;

(iii) for at least one resolution $\left(X, \mathcal{G}_{X}\right) \boxminus\left(Y_{x}, \mathcal{G}_{x}\right)_{x \in X}$ equal to $\left(Z, \mathcal{G}_{Z}\right)$, if $S$ coincides with a fiber $Y_{x}$, then $x \in \operatorname{ex}_{\mathcal{G}_{X}}(X)$;

(iv) $Z \backslash S \in \mathcal{G}_{Z}$.

Proof. We prove (i) $\Longrightarrow$ (iv) $\Longrightarrow$ (ii) $\Longrightarrow$ (i), and leave the (simple) proof of (ii) $\Longrightarrow$ (iii) $\Longrightarrow$ (iv) to the reader. Let $T:=Z \backslash S$, where $S$ is shrinkable in $Z$.

(i) $\Longrightarrow$ (iv). If $S$ is extremely shrinkable in $\left(Z, \mathcal{G}_{Z}\right)$, then by definition there is an extreme resolution $\left(X, \mathcal{G}_{X}\right) \boxminus\left(Y_{x}, \mathcal{G}_{x}\right)_{x \in X}$ equal to $\left(Z, \mathcal{G}_{Z}\right)$ such that $S=Y_{x}$ for some $x \in X$. Since $|S| \geqslant 2$, the hypothesis yields $x \in \operatorname{ex}_{\mathcal{G}_{x}}(X)$. We claim that $T \in \mathcal{G}_{Z}$. Requirement (R1) in Definition 19 holds for $T$ by the equivalence (2) in Remark 22. Next, as $T \cap Y_{x}=\varnothing$ and $T \cap Y_{x^{\prime}}=Y_{x^{\prime}}$ for $x^{\prime} \in X \backslash\{x\}$, we derive that (R2) holds for $T$ as well. Since $T$ also satisfies (R3), the implication (i) $\Longrightarrow$ (iv) is fully proved.

(iv) $\Longrightarrow$ (ii). Suppose $T \in \mathcal{G}_{Z}$. In the resolution considered in (ii), the assumption implies $\pi(T)=X \backslash\{x\} \in \mathcal{G}_{X}$. It follows that $x \in \operatorname{ex}_{\mathcal{G}_{x}}(X)$.

(ii) $\Longrightarrow$ (i). Suppose (ii) holds. As $S$ is assumed to be shrinkable, there exists (as in the proof of Theorem 26) a resolution in which one fiber equals $S$ and all the other fibers have size 1. By (ii), the projection of $S$ is an extreme element of the base. As a consequence, the resolution is extreme.

A characterization of shrinkable subsets in terms of convex sets appeared in Theorems 26 . We derive a simpler characterization for extremely shrinkable sets.

Theorem 36. Let $\left(Z, \mathcal{G}_{Z}\right)$ be a convex geometry. The following statements are equivalent for a subset $S$ of $Z$ such that $1<|S|<|Z|$ :

(i) $S$ is extremely shrinkable;

(ii) $S$ satisfies the following properties for any $G, H \in \mathcal{G}_{Z}$ :

(V1) $|G \cap S|=1 \Longrightarrow G \cup S \in \mathcal{G}_{Z}$;

(V2) $(Z \backslash S) \cup(H \cap S) \in \mathcal{G}_{Z}$. 
Proof. (i) $\Longrightarrow$ (ii). Assume $S$ is extremely shrinkable. To prove (V1), suppose $G \in \mathcal{G}_{Z}$ and $|G \cap S|=1$. By Theorem 35, the hypothesis entails $Z \backslash S \in \mathcal{G}_{Z}$, whence $G \backslash S=$ $G \cap(Z \backslash S) \in \mathcal{G}_{Z}$. Since $S$ is shrinkable, Property (S2) from Theorem 26 yields (taking $H:=Z) G \cup S \in \mathcal{G}_{Z}$. To prove (V2), let $H \in \mathcal{G}_{Z}$. Then, for $G:=Z$ in (S2), we get $(Z \backslash S) \cup(H \cap S) \in \mathcal{G}_{Z}$, as desired.

(ii) $\Longrightarrow$ (i). Assume (V1) and (V2) hold. We first derive the shrinkability of $S$ by establishing Properties (S1) and (S2) in Theorem 26. Property (S1) holds because $G \backslash S=G \cap(Z \backslash S) \in \mathcal{G}_{Z}$ follows from (V2) with $H=\varnothing$. To prove (S2), let $G, H \in \mathcal{G}_{Z}$ be such that $G \cap S \neq \varnothing$ and $G \backslash S \in \mathcal{G}_{Z}$. In view of Remark 27, we can assume $|G \cap S|=1$. Using

$$
(G \backslash S) \cup(H \cap S)=(G \cup S) \cap((Z \backslash S) \cup(H \cap S)),
$$

we derive from both (V1) and (V2) that the latter set lies in $\mathcal{G}_{Z}$. This proves that $S$ is shrinkable. Finally, observe that $S$ is also extremely shrinkable, because $Z \backslash S \in \mathcal{G}_{Z}$ follows from (V2) with $H:=\varnothing$, hence we can make use of Theorem 35.

Remark 37. In case an extremely shrinkable set $S$ is convex in $\mathcal{G}_{Z}$, Property (V2) becomes equivalent to the following one:

$$
\left(\mathrm{V}^{\prime}\right) \quad\left(H \in \mathcal{G}_{Z} \wedge H^{\prime} \subseteq S\right) \Longrightarrow(Z \backslash S) \cup H^{\prime} \in \mathcal{G}_{Z} .
$$

The reason is that $\left\{H \cap S \mid H \in \mathcal{G}_{Z}\right\}=\left\{H^{\prime} \in \mathcal{G}_{Z} \mid H^{\prime} \subseteq S\right\}$ whenever $S \in \mathcal{G}_{Z}$.

\subsection{Resolutions and Homomorphisms of Semilattices}

This subsection is a brief detour from the main topic, and it is not needed to read the remainder of the paper. However, it becomes important in view of possible developments, because it establishes a connection between resolutions of convex geometries and homomorphisms of certain semilattices. We thank an anonymous referee for pointing out the relevance of semilattice homomorphisms, and making several important remarks.

Let $\left(X, \mathcal{G}_{X}\right)$ be a convex geometry. The poset $\left(\mathcal{G}_{X}, \subseteq\right)$ is a join-semilattice, ${ }^{8}$ in which the join $G \vee H$ of any two elements $G, H \in \mathcal{G}_{X}$ is equal to $\operatorname{conv}_{\mathcal{G}_{X}}(G \cup H)$. Although $\left(\mathcal{G}_{X}, \subseteq\right)$ is also a lattice (in which the meet $G \wedge H$ of $G$ and $H$ is equal to $\left.G \cap H\right)$, here we are especially interested in the join-semilattice structure, because the canonical projection of a resolution yields an epimorphism of join-semilattices: ${ }^{9}$

Proposition 38. Let $\left(Z, \mathcal{G}_{Z}\right)=\left(X, \mathcal{G}_{X}\right) \boxminus\left(Y_{x}, \mathcal{G}_{x}\right)_{x \in X}$ be a resolution of convex geometries with projection $\pi: Z \rightarrow X$. The induced set-function $\pi: \mathcal{G}_{Z} \rightarrow \mathcal{G}_{X}$ is a homomorphism of the join-semilattice $\left(\mathcal{G}_{Z}, \subseteq\right)$ onto the join-semilattice $\left(\mathcal{G}_{X}, \subseteq\right)$, where homomorphism here means that the equality

$$
\pi(G \vee H)=\pi(G) \vee \pi(H)
$$

\footnotetext{
${ }^{8}$ For the terminology on semilattices, we recommend Grätzer (2011). For links with convex geometries, two main references are Monjardet (1990) and Adaricheva and Nation (2016b).

${ }^{9}$ We use the same notation $\vee$ and $\wedge$ for, respectively, the join and the meet in the two lattices $\left(\mathcal{G}_{Z}, \subseteq\right)$ and $\left(\mathcal{G}_{X}, \subseteq\right)$. An epimorphism is a surjective homomorphism.
} 
holds for all $G, H \in \mathcal{G}_{Z}$. On the contrary, $\pi$ may fail to be a lattice homomorphism, because $\pi(G \wedge H)=\pi(G) \wedge \pi(H)$ does not hold in all resolutions.

Proof. By Definition 19 of resolutions, the set-function $\pi$ is well-defined because $\pi(G) \in$ $\mathcal{G}_{X}$ for any $G \in \mathcal{G}_{Z}$. Furthermore, $\pi$ is surjective, because any convex set $A$ in $\mathcal{G}_{X}$ is the image by $\pi$ of the convex set $\{z \in Z \mid \pi(z) \in A\}$ in $\mathcal{G}_{Z}$.

Next, we show that $\pi$ is a join-semilattice homomorphism. To start, observe that an application of $\pi$ to both sides of Equation (3) in Lemma 23 yields for all $A \in 2^{Z}$

$$
\pi\left(\operatorname{conv}_{\mathcal{G}_{Z}}(A)\right)=\operatorname{ex}_{\mathcal{G}_{X}}(\pi(A)) \cup \operatorname{conv}_{\mathcal{G}_{X}}(\pi(A)) \backslash \operatorname{ex}_{\mathcal{G}_{X}}(\pi(A))=\operatorname{conv}_{\mathcal{G}_{X}}(\pi(A)) .
$$

Now apply the last equality to the set $A:=G \cup H$ to obtain

$$
\begin{aligned}
\pi(G \vee H) & =\pi\left(\operatorname{conv}_{\mathcal{G}_{Z}}(G \cup H)\right) \\
& =\operatorname{conv}_{\mathcal{G}_{X}}(\pi(G \cup H)) \\
& =\operatorname{conv}_{\mathcal{G}_{X}}(\pi(G) \cup \pi(H)) \\
& =\pi(G) \vee \pi(H) .
\end{aligned}
$$

This proves that $\pi: \mathcal{G}_{Z} \rightarrow \mathcal{G}_{X}$ is an epimorphism of join-semilattices.

Finally, we show that $\pi$ may fail to preserve meet. Suppose there are two disjoint convex sets $K, L \in \mathcal{G}_{x}$ for some $x \in X$. Set

$$
A:=\operatorname{conv}_{\mathcal{G}_{X}}(\{x\}), \quad G:=K \cup \bigcup_{y \in A \backslash\{x\}} Y_{y}, \quad H:=L \cup \bigcup_{y \in A \backslash\{x\}} Y_{y} .
$$

Note that $G, H \in \mathcal{G}_{Z}$ and $Y_{x} \cap G \cap H=\varnothing$, whence $x \notin \pi(G \wedge H)=\pi(G \cap H)$. Since $x \in \pi(G) \cap \pi(H)=\pi(G) \wedge \pi(H), \pi$ is not a meet-semilattice homomorphism.

A join-semilattice $\left(S, \leqslant_{S}\right)$ is the join-semilattice of some convex geometry if and only if $\left(S, \leqslant_{S}\right)$ is 'graded', with height one more than the number of the 'join-irreducible' elements (see definition in next paragraph). This characterization as well as several other ones are detailed in Monjardet (1990), and Adaricheva and Nation (2016b).

As we now explain ${ }^{10}$ any convex geometry $\left(X, \mathcal{G}_{X}\right)$ is canonically recoverable from (the structure of) its join-semilattice $\left(\mathcal{G}_{X}, \subseteq\right)$. By definition, an element $J$ of $\left(\mathcal{G}_{X}, \subseteq\right)$ is join-irreducible when (i) $A \subsetneq J$ for some $A \in \mathcal{G}_{X}$, and (ii) for all $B, C \in \mathcal{G}_{X}$ the equality $J=B \vee C$ implies $J=B$ or $J=C$ (thus $J$ is join-irreducible if and only if it covers exactly one other element $\left.{ }^{11}\right)$. It is not difficult to check that a convex set $J$ in the convex geometry $\left(X, \mathcal{G}_{X}\right)$ is a join-irreducible element of the join-semilattice $\left(\mathcal{G}_{X}, \subseteq\right)$ if and only if $J=\operatorname{conv}_{\mathcal{G}_{X}}(\{x\})$ for some $x \in X$. We denote by $\operatorname{jir}\left(\mathcal{G}_{X}\right)$ the set of all joinirreducible elements of the join-semilattice $\left(\mathcal{G}_{X}, \subseteq\right)$ : see Figures 3 and 4 for examples of

\footnotetext{
${ }^{10}$ For the more general case of standard closure spaces, see Adaricheva and Nation (2016a, Theorem 4$2.14)$.

${ }^{11}$ In any ordered set $(E, \leqslant)$, the element $b$ covers the element $a$ when $a<b$ and there is no element $c$ such that $a<c<b$. Then $(a, b)$ is a covering pair.
} 
join-semilattices of convex geometries, with the join-irreducible elements shown as filledsquare nodes and their covering edges highlighted. This gives a bijection

$$
g: X \rightarrow \operatorname{jir}\left(\mathcal{G}_{X}\right): x \mapsto \operatorname{conv}_{\mathcal{G}_{x}}(\{x\})
$$

Next, we define the function

$$
h: \mathcal{G}_{X} \rightarrow 2^{\mathrm{jir}\left(\mathcal{G}_{X}\right)}: G \mapsto\left\{J \in \operatorname{jir}\left(\mathcal{G}_{X}\right) \mid J \subseteq G\right\} .
$$

This function is injective, because $h(G)=\{g(a) \mid a \in G\}$ for each $G \in \mathcal{G}_{X}$. To make $h$ bijective, we take its image $\mathcal{J}\left(\mathcal{G}_{X}\right):=h\left(\mathcal{G}_{X}\right)$ as codomain. Because for all $G \in \mathcal{G}_{X}$

$$
\begin{aligned}
G & =\operatorname{conv}_{\mathcal{G}_{x}}\left(\bigcup_{a \in G} \operatorname{conv}_{\mathcal{G}_{x}}(\{a\})\right) \\
& =\bigvee_{a \in G} \operatorname{conv}_{\mathcal{G}_{x}}(\{a\}) \\
& =\bigvee g(G),
\end{aligned}
$$

the image $\mathcal{J}\left(\mathcal{G}_{X}\right)$ of $h$ consists of the subsets $K$ of $\operatorname{jir}\left(\mathcal{G}_{X}\right)$ satisfying

$$
K=\left\{J \in \operatorname{jir}\left(\mathcal{G}_{X}\right) \mid J \subseteq \bigvee K\right\}
$$

To conclude: the canonical bijections $g$ and $h$ lead to identify, respectively $X$ with $\operatorname{jir}\left(\mathcal{G}_{X}\right)$, and $\mathcal{G}_{X}$ with $\mathcal{J}\left(\mathcal{G}_{X}\right)$. Moreover, for $x \in X$ and $G \in \mathcal{G}_{X}$, we have $x \in G$ exactly when $g(x) \in h(G)$, or equivalently $g(x) \subseteq G$.

Next, we similarly interpret resolutions of convex geometries in terms of certain homomorphisms. According to Proposition 38, the projection $\pi$ of any resolution $\left(Z, \mathcal{G}_{Z}\right)=$ $\left(X, \mathcal{G}_{X}\right) \boxminus\left(Y_{x}, \mathcal{G}_{x}\right)_{x \in X}$ induces a join-semilattice homomorphism $\pi: \mathcal{G}_{Z} \rightarrow \mathcal{G}_{X}$, defined by $G \mapsto \pi(G)$. Note that $\pi^{-1}(\varnothing)=\{\varnothing\}$; in Grätzer (2011) terms, the homomorphism $\pi$ is $\{0\}$-separating (when a join-semilattice has a minimum, the latter is often denoted as 0 ). Moreover, $\pi$ also maps $\operatorname{jir}\left(\mathcal{G}_{Z}\right)$ onto $\operatorname{jir}\left(\mathcal{G}_{X}\right)$.

Remark 39. A homomorphism of join-semilattices of convex geometries needs not map join-irreducible elements on join-irreducible elements. Figure 3 displays the Hasse diagrams of the join-semilattices $(\mathcal{S}, \subseteq)$ and $\left(\mathcal{S}^{\prime}, \subseteq\right)$ of two convex geometries. The function $\pi: \mathcal{S} \rightarrow \mathcal{S}^{\prime}$, defined by $K \mapsto K^{\prime}$, provides a counterexample. Indeed, it is a join homomorphism from $(\mathcal{S}, \subseteq)$ onto $\left(\mathcal{S}^{\prime}, \subseteq\right)$, and it maps the join-irreducible element $D$ to the join-reducible element $D^{\prime}$.

It would be interesting to characterize, for two given join-semilattices $\left(\mathcal{G}_{Z}, \subseteq\right)$ and $\left(\mathcal{G}_{X}, \subseteq\right)$ of convex geometries, when a function $\pi: \mathcal{G}_{Z} \rightarrow \mathcal{G}_{X}$ is induced by a resolution (the resolution has as result the first convex geometry and as base the second convex geometry). The condition that $\pi$ is a 0 -separating join-homomorphism mapping $\operatorname{jir}\left(\mathcal{G}_{Z}\right)$ onto $\operatorname{jir}\left(\mathcal{G}_{X}\right)$ is necessary but not sufficient, as next example shows. 

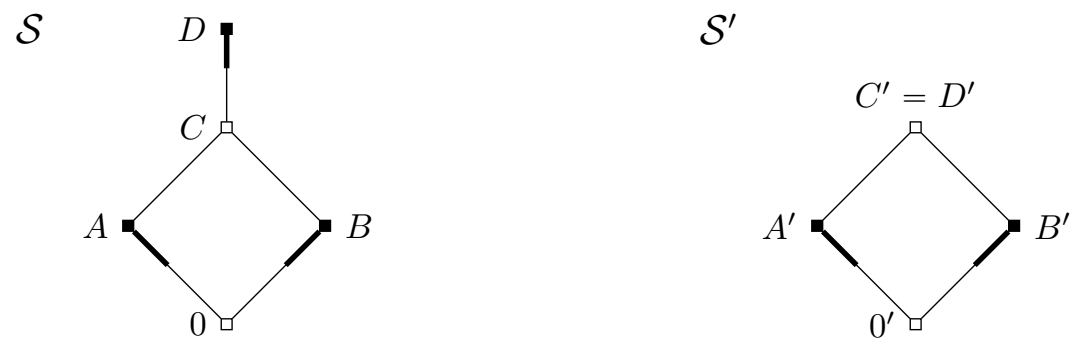

Figure 3: The homomorphism of join-semilattices of convex geometries used in Remark 39.

Example 40. The left part of Figure 4 shows a join-semilattice communicated by a referee, which is the join-semilattice $\left(\mathcal{G}_{Z}, \subseteq\right)$ of a convex geometry $\left(Z, \mathcal{G}_{Z}\right)$ on 4 elements. On the right, the figure displays the join-semilattice $\left(\mathcal{G}_{X}, \subseteq\right)$ of another convex geometry $\left(X, \mathcal{G}_{X}\right)$. The function described in the middle of the figure is a join-semilattice homomorphism, which is 0 -separating and maps $\operatorname{jir}\left(\mathcal{G}_{Z}\right)$ onto $\operatorname{jir}\left(\mathcal{G}_{X}\right)$. However, there is no resolution with base $\left(X, \mathcal{G}_{X}\right)$ producing $\left(Z, \mathcal{G}_{Z}\right)$. Indeed, $\left(Z, \mathcal{G}_{Z}\right)$ is a primitive (or irresolvable) convex geometry: it has no shrinkable set, as seen through a (bit tedious) application of Theorem 26.

As we just noted, the convex geometry $\left(Z, \mathcal{G}_{Z}\right)$ in the left of Figure 4 is primitive. The referee considers that $\left(Z, \mathcal{G}_{Z}\right)$ is 'decomposable'. Therefore, two problems (which are left for future work) arise: first, to characterize resolutions of convex geometries in terms of join-semilattice homomorphisms; second, to understand the other type of decomposability pointed out by the referee. Of course, to answer the first problem, we could just mimic the definition of resolutions in terms of the join-semilattice homomorphism produced by the projection (recovering each convex geometry on the set of join-irreducible elements of the join-semilattice of the convex geometry); however, what we are asking here is an answer which is truly at the level of join-semilattice homomorphisms.

\section{Resolutions of Special Convex Geometries}

Here we examine resolutions of the two classes of convex geometries introduced in Section 2.1: ordinal and affine. We start with the affine case.

\subsection{Affine Convex Geometries}

Recall from Example 6 that a convex geometry is affine if and only if it is isomorphic to a convex geometry induced on a finite subset of a real affine space.

Lemma 41. If a resolution is an affine convex geometry, then the base and the fibers of the resolution are also affine convex geometries.

Proof. The result follows at once from Remark 22: the fibers are subgeometries of the resolution, and the base is isomorphic to a subgeometry of the resolution. 


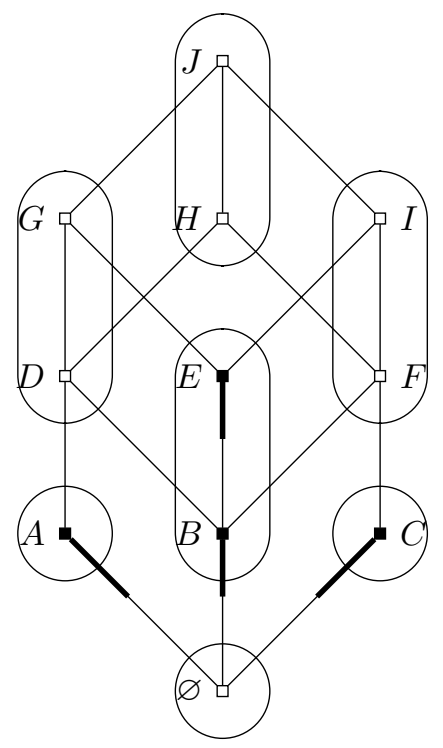

$$
\begin{aligned}
J & \mapsto Q \\
G & \mapsto N \\
H & \mapsto Q \\
I & \mapsto P \\
D & \mapsto N \\
E & \mapsto L \\
F & \mapsto P \\
A & \mapsto K \\
B & \mapsto L \\
C & \mapsto M \\
\varnothing & \mapsto \varnothing
\end{aligned}
$$

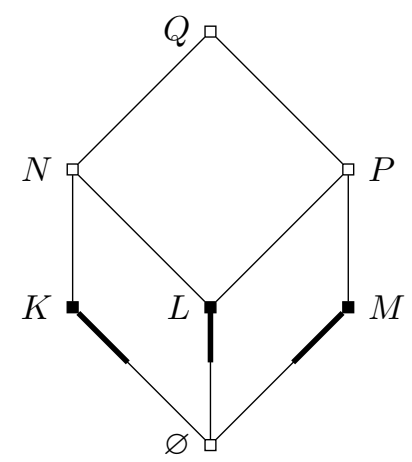

Figure 4: See Example 40. On the left, the Hasse diagram of the join-semilattice of a convex geometry communicated by a referee (we have added a representation of the preimages of the join homomorphism - which are thus the equivalence classes of a "join-semilattice congruence'). In the middle, the specification of the join-semilattice homomorphism. On the right, the join-semilattice (of a convex geometry) that is the image of this homomorphism.

The converse of Lemma 41 does not hold: Examples 42 and 50 below show that resolutions of affine convex geometries need not be affine.

Example 42. Let

$$
\begin{array}{ll}
X=\{1,2,3\}, & \mathcal{G}_{X}=2^{X} \backslash\{\{1,3\}\}, \\
Y_{1}=\{a\}, & \mathcal{G}_{1}=2^{Y_{1}}, \\
Y_{2}=\{b, c\}, & \mathcal{G}_{2}=2^{Y_{2}} \\
Y_{3}=\{d\}, & \mathcal{G}_{3}=2^{Y_{3}} .
\end{array}
$$

All pairs $\left(X, \mathcal{G}_{X}\right)$ and $\left(Y_{i}, \mathcal{G}_{i}\right)$, for $i=1,2,3$, are affine convex geometries. A simple computation shows that the resolution of $\left(X, \mathcal{G}_{X}\right)$ into $\left\{\left(Y_{i}, \mathcal{G}_{i}\right) \mid i \in X\right\}$ is the convex geometry $\left(Z, \mathcal{G}_{Z}\right)=\left(X, \mathcal{G}_{X}\right) \boxminus\left(Y_{i}, \mathcal{G}_{i}\right)_{i \in X}$, where $Z=\{a, b, c, d\}$, and

$$
\mathcal{G}_{Z}=2^{Z} \backslash\{\{a, d\},\{a, b, d\},\{a, c, d\}\} .
$$

(See Figure 5, which describes the lattice $\left(\mathcal{G}_{Z}, \subseteq\right)$ of convex sets.) However, the convex geometry $\left(Z, \mathcal{G}_{Z}\right)$ is not affine. This readily follows from the fact that $b, c \in \operatorname{conv}_{\mathcal{G}_{Z}}(\{a, d\})$, but $b \notin \operatorname{conv}_{\mathcal{G}_{Z}}(\{a, c\})$ and $c \notin \operatorname{conv}_{\mathcal{G}_{Z}}\left(\{a, b\}\right.$ : indeed, if $\left(Z, \mathcal{G}_{Z}\right)$ were affinely embedded in some real affine space, we would have $b$ and $c$ on the segment $[a, d]$, and so either $b \in[a, c]$ or $c \in[a, b]$. 


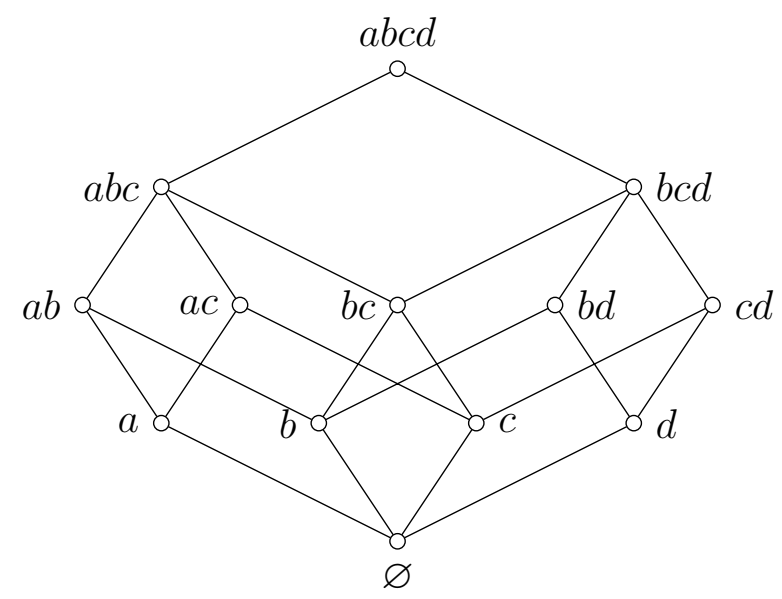

Figure 5: The lattice of convex sets of the (non-affine) resolution in Example 42.

Observe that the non-affine resolution $\left(Z, \mathcal{G}_{Z}\right)$ of Example 42 is also non-extreme, since $2 \notin \operatorname{ex}_{\mathcal{G}_{X}}(X)$ and yet $\left|Y_{2}\right|>1$. In fact, to conclude that $\left(Z, G_{Z}\right)$ is non-affine, it suffices to check that it is non-extreme, as the next result guarantees.

Theorem 43. An affine resolution of convex geometries is extreme.

Theorem 43 is a special case of a more general result (Theorem 44), which shows that Axiom (EA) below is sufficient for a resolution to be extreme. A convex geometry $\left(Z, \mathcal{G}_{Z}\right)$ satisfies Axiom (EA) when, for any $A \in 2^{Z}$ and $p \in Z$,

$$
\text { (EA) } p \in \operatorname{conv}_{\mathcal{G}_{Z}}(A) \Longrightarrow \operatorname{conv}_{\mathcal{G}_{Z}}(A) \subseteq \bigcup_{a \in A} \operatorname{conv}_{\mathcal{G}_{Z}}((A \backslash\{a\}) \cup\{p\}) .
$$

Levi (1951) formulated this condition for 'closure spaces' (named (C3)). Sierksma (1984) calls it the 'exchange axiom'. ${ }^{12}$ In terms of Adaricheva and Nation (2016c), it is the ' $n$-carousel rule' restricted to $n=|X|-2$. Theorem 43 follows from Theorem 44, because any affine convex geometry satisfies (EA).

Theorem 44. A resolution of convex geometries satisfying Axiom (EA) is extreme.

Proof. Let $\left(Z, \mathcal{G}_{Z}\right)=\left(X, \mathcal{G}_{X}\right) \boxminus\left(Y_{x}, \mathcal{G}_{x}\right)_{x \in X}$ be a resolution of convex geometries which satisfies Axiom (EA). Denote by $E$ the set of all extreme points of the base set $X$. Toward a contradiction, assume there is $w \in X \backslash E$ such that $\left|Y_{w}\right| \geqslant 2$, and let $a, b$ be two distinct elements of $Y_{w}$. Since $w \in X=\operatorname{conv}_{\mathcal{G}_{X}}(E)$, there is a minimal subset $V$ of $E$ such that $w \in \operatorname{conv}_{\mathcal{G}_{X}}(V)$. For each $v \in V$, denote by $V_{v \leftarrow w}$ the set $(V \backslash\{v\}) \cup\{w\}$. By the minimality of $V$, we have

$$
w \in \operatorname{ex}_{\mathcal{G}_{X}}\left(V_{v \leftarrow w}\right) \text { for all } v \in V .
$$

\footnotetext{
${ }^{12}$ Not to be confused with the 'exchange condition' for matroids.
} 
Now let $T \subseteq Z$ be a transversal for the family $\left\{Y_{v} \mid v \in V\right\}$, that is, $\left|T \cap Y_{v}\right|=1$ for each $v$ in $V$. Observe that $\operatorname{ex}_{\mathcal{G}_{X}}(\pi(T))=\operatorname{ex}_{\mathcal{G}_{X}}(V)=V$ by Lemma 12(i). Therefore, Lemma 23 yields

$$
\operatorname{conv}_{\mathcal{G}_{Z}}(T)=\bigcup_{v \in V} \operatorname{conv}_{\mathcal{G}_{v}}\left(T \cap Y_{v}\right) \cup \bigcup_{x \in \operatorname{conv}_{\mathcal{G}_{X}}(V) \backslash V} Y_{x}
$$

and so, since $w \in \operatorname{conv}_{\mathcal{G}_{X}}(V) \backslash V$ and $a, b \in Y_{w}$, we deduce

$$
a, b \in \operatorname{conv}_{\mathcal{G}_{Z}}(T) .
$$

Since $a \in \operatorname{conv}_{\mathcal{G}_{Z}}(T)$, Axiom (EA) entails

$$
\operatorname{conv}_{\mathcal{G}_{Z}}(T) \subseteq \bigcup_{t \in T} \operatorname{conv}_{\mathcal{G}_{Z}}\left(T_{t \leftarrow a}\right)
$$

where $T_{t \leftarrow a}$ stands for $(T \backslash\{t\}) \cup\{a\}$. Since $b \in \operatorname{conv}_{\mathcal{G}_{Z}}(T)$, it follows

$$
b \in \operatorname{conv}_{\mathcal{G}_{Z}}\left(T_{u \leftarrow a}\right) \text { for some } u \in T .
$$

Let $r=\pi(u)$. Another application of Lemma 23 yields

$$
\operatorname{conv}_{\mathcal{G}_{Z}}\left(T_{u \leftarrow a}\right)=\bigcup_{x \in \operatorname{ex}_{\mathcal{G}_{X}}\left(V_{r \leftarrow w}\right)} \operatorname{conv}_{\mathcal{G}_{x}}\left(T_{u \leftarrow a} \cap Y_{x}\right) \cup \underset{x \in \operatorname{conv}_{\mathcal{G}_{X}}\left(V_{r \leftarrow w}\right) \backslash \operatorname{ex}_{\mathcal{G}_{X}}\left(V_{r \leftarrow w}\right)}{\bigcup_{x}}
$$

whence, by (13) and (15), we deduce

$$
b \in \operatorname{conv}_{\mathcal{G}_{w}}\left(T_{u \leftarrow a} \cap Y_{w}\right)=\operatorname{conv}_{\mathcal{G}_{w}}(\{a\}),
$$

which in turn implies $b \notin \operatorname{ex}_{\mathcal{G}_{w}}(\{a, b\})$. The roles of $a$ and $b$ being exchangeable, we also have $a \notin \operatorname{ex}_{\mathcal{G}_{w}}(\{a, b\})$. It follows that $\operatorname{ex}_{\mathcal{G}_{w}}(\{a, b\})=\varnothing$, a contradiction.

Corollary 45. Let $\left(Z, \mathcal{G}_{Z}\right)$ be an affine convex geometry. For any shrinkable set $S \subseteq Z$, we have:

(i) $S \in \mathcal{G}_{Z}$;

(ii) $G \backslash S \in \mathcal{G}_{Z}$ for all $G \in \mathcal{G}_{Z}$, in particular $Z \backslash S \in \mathcal{G}_{Z}$.

Proof. Fix $S \subseteq Z$ shrinkable. Thus there is a resolution $\left(X, \mathcal{G}_{X}\right) \boxminus\left(Y_{x}, \mathcal{G}_{x}\right)_{x \in X}$ producing $\left(Z, \mathcal{G}_{Z}\right)$ in which $S$ is a (nontrivial) fiber $Y_{x}$.

(i) By Lemma 41 , the base $\left(X, \mathcal{G}_{X}\right)$ is an affine convex geometry, which implies that any one-element set $\{x\}$ in $2^{X}$ belongs to $\mathcal{G}_{X}$. By (1) in Remark 22, we conclude $S=Y_{x} \in \mathcal{G}_{Z}$.

(ii) To start, we prove $Z \backslash S \in \mathcal{G}_{Z}$. By Theorem 43 , the resolution $\left(Z, \mathcal{G}_{Z}\right)=\left(X, \mathcal{G}_{X}\right) \boxminus$ $\left(Y_{x}, \mathcal{G}_{x}\right)_{x \in X}$ is extreme, and so $S=Y_{x}$ is extremely shrinkable. An application of Theorem 35 readily yields $Z \backslash S \in \mathcal{G}_{Z}$. Then, for arbitrary $G \in \mathcal{G}_{Z}$, we get $G \backslash S \in \mathcal{G}_{Z}$, because $G \backslash S=G \cap(Z \backslash S)$. 
Finally, we obtain a characterization of all affine convex geometries that are resolvable (hence of primitive ones):

Corollary 46. The following statements are equivalent for an affine convex geometry $\left(Z, \mathcal{G}_{Z}\right)$ :

(1) $\left(Z, \mathcal{G}_{Z}\right)$ is resolvable;

(2) there is a shrinkable subset $S$ of $Z$;

(3) there is an extremely shrinkable subset $S$ of $Z$;

(4) there is a subset $S$ of $Z$, with $1<|S|<|X|$, satisfying the following properties for any $G, H \in \mathcal{G}_{Z}$ :

$$
\begin{array}{ll}
\text { (V1) } & \left(G \in \mathcal{G}_{Z} \wedge|G \cap S|=1\right) \Longrightarrow G \cup S \in \mathcal{G}_{Z}, \\
(\mathrm{~V} 2) & H \in \mathcal{G}_{Z} \Longrightarrow(Z \backslash S) \cup(H \cap S) \in \mathcal{G}_{Z} .
\end{array}
$$

Proof. Simply observe that as a consequence of Theorem 43, affine resolutions fall under the application of Theorem 36.

The primitivity of a convex geometry is characterized by the non-existence of a shrinkable set. However, even for affine convex geometries, this characterization is not computationally effective, because Properties (V1) and (V2) are to be checked for all convex sets of the given geometry. We wonder whether there are more instructive answers to the next two, related problems.

Problem 47. Given an affine convex geometry $(Z, \mathcal{G})$, characterize when a subset of $Z$ is shrinkable.

Problem 48. Geometrically characterize when an affine convex geometry is primitive.

By Theorems 43 and 32, Problems 47 and 48 are also problems about compositions of affine convex geometries. Although they appear to be central problems, we were unable to find any mention of them in the literature.

The next result states an equivalent (geometric) formulation of Property (T1) in Theorem 29. This reformulation is in the spirit of the answers we would like to obtain for Problems 47 and 48. In what follows, ' $\operatorname{conv}_{\mathcal{G}_{Z}}$ ' denotes the convex hull operator in a convex geometry $\mathcal{G}_{Z}$, whereas ' $\operatorname{conv}_{\mathbb{R}}$ ' is used for the standard convex hull in the affine space $\mathbb{R}^{d}$.

Proposition 49. Let $Z$ be a finite subset of $\mathbb{R}^{d}$, and $(Z, \mathcal{G})$ the convex geometry induced on $Z$. The following statements are equivalent for any set $S \subseteq Z$ such that $1<|S|<|Z|$ :

(i) S satisfies Property (T1) in Theorem 29, namely for all $A \in 2^{Z}$,

$$
(\mathrm{T} 1) \operatorname{ex}(A) \cap S \neq \varnothing \Longrightarrow \operatorname{ex}(A \cap S) \subseteq \operatorname{ex}(A) ;
$$


(ii) there exist proper faces $F_{1}, F_{2}, \ldots, F_{k}(k \geqslant 1)$ of the convex polytope $\operatorname{conv}_{\mathbb{R}}(Z)$ such that $S=Z \cap\left(F_{1} \cup F_{2} \cup \cdots \cup F_{k}\right)$.

A simple consequence of Condition (ii) is that $S$ lies in the relative boundary ${ }^{13}$ of $\operatorname{conv}_{\mathbb{R}}(Z)$ However, Condition (ii) asserts more than that.

Proof. Fix $S \subseteq Z$ such that $1<|S|<|Z|$.

(ii) $\Longrightarrow$ (i): Suppose there exist proper faces $F_{1}, F_{2}, \ldots, F_{k}$ of the convex polytope $\operatorname{conv}_{\mathbb{R}}(Z)$ such that $S=Z \cap\left(F_{1} \cup F_{2} \cup \cdots \cup F_{k}\right)$. If any face $F_{i}$ equals $\operatorname{conv}_{\mathbb{R}}(Z)$, then $S=Z$, and so $S$ satisfies (T1). Thus we may assume that all $F_{i}$ 's are proper faces of $\operatorname{conv}_{\mathbb{R}}(Z)$. Let $A \in 2^{Z}$; we shall show that $\operatorname{ex}(A \cap S) \subseteq \operatorname{ex}(A)$. Given $w$ in $\operatorname{ex}(A \cap S)$, we know by (ii) that $w$ belongs to some face $F_{i}$ of $\operatorname{conv}_{\mathbb{R}}(Z)$, with moreover $Z \cap F_{i} \subseteq S$. If $w \in \operatorname{ex}(A)$ does not hold, then there exists a subset $B$ of $A \backslash\{w\}$ such that $w \in \operatorname{conv}_{\mathbb{R}}(B)$. Such a minimal subset $B$ of $A \backslash\{w\}$ is formed by the vertices $b_{1}, b_{2}, \ldots, b_{\ell}$ of a simplex containing $w$ in its relative interior. Then all $b_{j}$ 's belong to $F_{i}$, because the proper face $F_{i}$ of $\operatorname{conv}_{\mathbb{R}}$ equals the intersection of $\operatorname{conv}_{\mathbb{R}}(Z)$ with some hyperplane supporting $\operatorname{conv}_{\mathbb{R}}(Z)$. It follows that, for all $j$ 's, we have $b_{j} \in A \cap F_{i} \subseteq A \cap Z \cap F_{i} \subseteq A \cap S$, contradicting the initial assumption $w \in \operatorname{ex}(A \cap S)$.

(i) $\Longrightarrow$ (ii): Suppose $S$ satisfies Property (T1).

Claim: If some point $w$ of $S$ is in the relative interior of any face $F$ (proper or not) of $\operatorname{conv}_{\mathbb{R}}(Z)$, then $Z \cap F \subseteq S$ and the face $F$ is proper.

Proof of Claim. Toward a contradiction, assume there is $f_{0} \in(Z \cap F) \backslash S$. The line passing through $f_{0}$ and $w$ must meet the relative boundary of $F$ on the side of $w$ opposite to $f_{0}$. Thus there exist vertices $f_{1}, f_{2}, \ldots, f_{k}$ of the face $F$ such that $w$ belongs to the relative interior of the simplex with vertices $f_{0}, f_{1}, \ldots, f_{k}$. Notice $\left\{f_{0}, f_{1}, f_{2}, \ldots, f_{k}\right\} \subseteq Z$ (because all vertices of $\operatorname{conv}_{\mathbb{R}}(Z)$ must be in $Z$ ). We split the analysis in the only two possible cases.

Case 1: $f_{i}$ is in $S$, for some $i \in\{1,2, \ldots, k\}$. Set $A=\left\{w, f_{0}, f_{1}, \ldots, f_{k}\right\}$, and notice $f_{i} \in \operatorname{ex}(A) \cap S$ together with $w \in \operatorname{ex}(A \cap S) \backslash \operatorname{ex}(A)$. This contradicts the assumption that $S$ satisfies (T1).

Case 2: $\left\{f_{0}, f_{1}, \ldots, f_{k}\right\} \subseteq Z \backslash S$. By our assumption $|S| \geqslant 2$, there is $v \in S \backslash\{w\}$. Consider two subcases for the possible position of the point $v$. First, if $v \notin \operatorname{conv}_{\mathbb{R}}\left(\left\{f_{0}, f_{1}\right.\right.$, $\left.\left.f_{2}, \ldots, f_{k}\right\}\right)$, then we set $A=\left\{v, w, f_{0}, f_{1}, f_{2}, \ldots, f_{k}\right\}$. Notice $v \in \operatorname{ex}(A) \cap S$ and $w \in \operatorname{ex}(A \cap S) \backslash \operatorname{ex}(A)$, again a contradiction with $S$ satisfying (T1). Second, if $v \in \operatorname{conv}_{\mathbb{R}}\left(\left\{f_{0}, f_{1}, f_{2}, \ldots, f_{k}\right\}\right)$, there is a point $x$ on the relative boundary of the simplex $T=\operatorname{conv}_{\mathbb{R}}\left(\left\{f_{0}, f_{1}, \ldots, f_{k}\right\}\right)$ such that $\left.w \in\right] v, x[$. Let now $A$ be formed by the points $v, w$ and the vertices of the minimal face of the simplex $T$ which contains $x$. Again we get a contradiction because $v \in \operatorname{ex}(A) \cap S$ and $w \in \operatorname{ex}(A \cap S) \backslash \operatorname{ex}(A)$.

To complete the proof of the Claim, simply observe that $F$ must be proper, because otherwise we would have $Z=S$.

\footnotetext{
${ }^{13}$ That is, the boundary computed in the affine subspace generated by $\operatorname{conv}_{\mathbb{R}}(Z)$.
} 
From the Claim, we derive that $S$ contains the intersection of $Z$ with any face of $\operatorname{conv}_{\mathbb{R}}(Z)$ containing in its relative interior at least one point of $S$. Thus $S$ includes the intersection of $Z$ with the union of all such faces. The reverse inclusion also holds, because any point $w$ of $S$ belongs to both $Z$ and the relative interior of the smallest face of $\operatorname{conv}_{\mathbb{R}}(Z)$ containing $w$.

We are still missing a translation of Property (T2) in Theorem 29. This translation appears to be of some interest in view of the fact that, along with the translation of (T1) obtained in Proposition 49, it would deliver a solution to Problem 47.

The next example illustrates another type of obstruction to the affineness of a resolution.

Example 50. Let

$$
\begin{array}{ll}
X=\{1,2,3\}, & \mathcal{G}_{X}=2^{X} \backslash\{\{1,3\}\}, \\
Y_{1}=\{a\}, & \mathcal{G}_{1}=2^{Y_{1}}, \\
Y_{2}=\{b\}, & \mathcal{G}_{2}=2^{Y_{2}} \\
Y_{3}=\{c, d\}, & \mathcal{G}_{1}=2^{Y_{3}} .
\end{array}
$$

The resolution of $\left(X, \mathcal{G}_{X}\right)$ into $\left\{\left(Y_{i}, \mathcal{G}_{i}\right) \mid i \in X\right\}$ is the convex geometry $\left(Z, \mathcal{G}_{Z}\right)=\left(X, \mathcal{G}_{X}\right)$ 日 $\left(Y_{i}, \mathcal{G}_{i}\right)_{i \in X}$, where $Z=\{a, b, c, d\}$, and

$$
\mathcal{G}_{Z}=2^{Z} \backslash\{\{a, c\},\{a, d\}\} .
$$

Although all convex geometries $\left(X, \mathcal{G}_{X}\right)$ and $\left(Y_{i}, \mathcal{G}_{i}\right), i=1,2,3$, are affine, their resolution $\left(Z, \mathcal{G}_{Z}\right)$ is not. Indeed, we have $b \in \operatorname{conv}_{\mathcal{G}_{Z}}(\{a, c\}) \cap \operatorname{conv}_{\mathcal{G}_{Z}}(\{a, d\})$, along with $c \notin \operatorname{conv}_{\mathcal{G}_{Z}}\left(\{b, d\}\right.$ and $d \notin \operatorname{conv}_{\mathcal{G}_{Z}}(\{b, c\}$, which is impossible in any affine geometry. (If $\left(Z, \mathcal{G}_{Z}\right)$ were affinely embedded, we would have in some real affine space $c$ and $d$ on the line through $a$ and $b$, on the side of $b$ opposite to $a$. However, this implies $c \in[b, d]$ or $d \in[b, c]$.

The crucial assumptions in the last example are that 2 lies between 1 and 3, and that the fiber $Y_{3}$ contains more than one element. We generalize them in the next proposition (where $p$ plays the role of 2 ).

Proposition 51. Suppose a resolution $\left(Z, \mathcal{G}_{Z}\right)=\left(X, \mathcal{G}_{X}\right) \boxminus\left(Y_{x}, \mathcal{G}_{x}\right)_{x \in X}$ of convex geometries is affine, with $\mathcal{G}_{Z}$ the geometry induced on the subset $Z$ of some real affine space $\mathbb{R}^{d}$. Assume that the base contains elements $p, p_{1}, \ldots, p_{n+1}$ such that $p \in \operatorname{conv}_{\mathcal{G}_{X}}\left(\left\{p_{1}\right.\right.$, $\left.\left.p_{2}, \ldots, p_{n+1}\right\}\right)$ and $p \notin \operatorname{conv}_{\mathcal{G}_{X}}(T)$ for any proper subset $T$ of $\left\{p_{1}, p_{2}, \ldots, p_{n+1}\right\}$. For $i=1,2, \ldots, n+1$, let $q_{i}$ be any point in the fiber $Y_{p_{i}}$. Then all fibers $Y_{p_{i}}$ lie in the affine subspace of dimension $n$ generated by the points $q_{1}, q_{2}, \ldots, q_{n+1}$, and so all fibers $\left(Y_{p_{i}}, \mathcal{G}_{p_{i}}\right)$ are isomorphic to convex geometries affinely embedded in a real affine space of dimension $n$.

Proof. As mentioned in Lemma 41 , the base $\left(X, \mathcal{G}_{X}\right)$ and all fibers $\left(Y_{x}, \mathcal{G}_{x}\right)$ are also affine geometries. By Theorem 43, the fiber $Y_{p}$ contains just one point, say $q$. Note that $q \in \operatorname{conv}_{\mathbb{R}}\left(\left\{q_{1}, q_{2}, \ldots, q_{n+1}\right\}\right)$ in $\mathbb{R}^{d}$ (the reason is that the projection on the base of the 
convex hull $\operatorname{conv}_{\mathcal{G}_{Z}}\left\{q_{1}, q_{2}, \ldots, q_{n+1}\right\}$ in $Z$ must be convex in the base $X$ and at the same time contain $p_{1}, p_{2}, \ldots, p_{n+1}$; thus by our assumptions the projection contains also $p$ ). Moreover, $q$ cannot be in the convex hull of less than $n+1$ of the points $q_{1}, q_{2}, \ldots, q_{n+1}$ (because the projection $p$ of $q$ does not lie in the convex hull in $X$ of less than $n+1$ of the $p_{i}$ 's). Thus in $\mathbb{R}^{d}$, the point $q$ is in the relative interior of the simplex with vertices $q_{1}, q_{2}$, $\ldots, q_{n+1}$. To derive the thesis for $i=1$ (the arguments are similar for the other values of $i$ ), note that $q_{1}$ lies in the affine hull of the points $q, q_{2}, q_{3}, \ldots, q_{n}$. As this result holds for any point in the fiber $Y_{p_{1}}$ in place of $q_{1}$, we deduce that the fiber $Y_{p_{1}}$ is included in the affine hull of $q, q_{2}, q_{3}, \ldots, q_{n+1}$, which is the same as the affine hull of $q_{1}, q_{2}, q_{3}, \ldots$, $q_{n+1}$.

We know of several other necessary conditions for an affine convex geometry to be primitive, but none of them is both necessary and sufficient. We leave Problems 47 and 48 unsolved.

\subsection{Ordinal Convex Geometries}

Recall from Example 4 and Theorem 5 that a convex geometry $\left(Z, \mathcal{G}_{Z}\right)$ is ordinal if and only if $\mathcal{G}_{Z}$ is closed under union, or, equivalently, $\mathcal{G}_{Z}$ consists of all ideals of some unique partial order $\leqslant$ on $Z$ (the partial order associated to $\mathcal{G}_{Z}$ ). Remark 17 readily yields that the equivalence

$$
\neg\left(z<z^{\prime}\right) \quad \Longleftrightarrow \quad z \in \operatorname{ex}_{\mathcal{G}_{Z}}\left(\left\{z, z^{\prime}\right\}\right)
$$

holds for all $z, z^{\prime} \in Z$. Here we show that (1) a resolution of ordinal convex geometries is always ordinal, and (2) its associated partial order is the 'resolution' (as in the next definition) of the partial orders associated to the base and the fibers.

Definition 52. Let $X$ be a finite base set, and $\left\{Y_{x} \mid x \in X\right\}$ a family of finite, pairwise disjoint fiber sets disjoint from the base set. Furthermore, let $R_{X}$ be a binary relation on $X$, and $R_{x}$ a binary relation on $Y_{x}$ for each $x \in X$. Set $Z:=\bigcup_{x \in X} Y_{x}$, and call projection the function $\pi: Z \rightarrow X$, with $\pi(z)=x$ when $z \in Y_{x}$. The resolution of $\left(X, R_{X}\right)$ into $\left\{\left(Y_{x}, R_{x}\right) \mid x \in X\right\}$ is the pair $\left(Z, R_{Z}\right)$, where $R_{Z}$ is the binary relation on $Z$ defined by

$$
z R_{Z} z^{\prime} \Longleftrightarrow \begin{cases}\text { either } & (\exists x \in X)\left(z, z^{\prime} \in Y_{x} \wedge z R_{x} z^{\prime}\right), \\ \text { or } \quad & \left(\exists x, x^{\prime} \in X\right)\left(x \neq x^{\prime} \wedge z \in Y_{x} \wedge z^{\prime} \in Y_{x^{\prime}} \wedge x R_{X} x^{\prime}\right)\end{cases}
$$

for all $z, z^{\prime} \in Z$. With a slight abuse of terminology, we shall also say that $R_{Z}$ is the resolution of $R_{X}$ into the family $\left\{R_{x} \mid x \in X\right\}$. We use a notation similar to the one employed for convex geometries, namely

$$
\left(Z, R_{Z}\right)=\left(X, R_{X}\right) \square\left(Y_{x}, R_{x}\right)_{x \in X}
$$

A binary relation on a finite set is primitive when it cannot be obtained as a nontrivial resolution of relations, and is resolvable otherwise. ${ }^{14}$

\footnotetext{
${ }^{14}$ As usual, nontrivial means that the base has more than one element, and there is at least a fiber that has more than one element.
} 
Remark 53. Resolutions of binary relations are well-known, often under a different name: see, for instance, Dörfler (1971). For the special case of partial orders, they are called sums by Hiraguchi (1951), lexicographic sums by Trotter (1992, page 24), and ordered sums by Harzheim (2005, page 85). Observe that Bang-Jensen and Gutin (2001, page 8) use the term 'composition' in place of 'resolution'. In this paper, we employ the term 'resolution' for binary relations not only to avoid confusion, but also in an attempt to use a common name for the codification of the same concept in different mathematical settings.

It is well-known that the resolution of binary relations is a partial order exactly when the base relations and the fiber relations are all partial orders: see Hiraguchi (1951), Trotter (1992), or Harzheim (2005). The main result of this section (Theorem 54) proves two things: (1) a resolution of convex geometries is ordinal if and only if so are its base and its fibers; (2) there is a tight connection between resolutions of ordinal convex geometries and resolutions of partial orders.

Theorem 54. A resolution of convex geometries is an ordinal convex geometry if and only if its base and all its fibers are ordinal convex geometries. Furthermore, the partial order associated to the resolved convex geometry is equal to the resolution of the partial order associated to the base into the family of partial orders associated to the fibers.

Proof. Let $\left(Z, \mathcal{G}_{Z}\right)=\left(X, \mathcal{G}_{X}\right) \boxminus\left(Y_{x}, \mathcal{G}_{x}\right)_{x \in X}$ be a resolution of convex geometries.

If the convex geometry $\left(Z, \mathcal{G}_{Z}\right)$ is ordinal, equivalently $\mathcal{G}_{Z}$ is closed under union (Theorem 5), Remark 22 implies that all geometries $\left(X, \mathcal{G}_{X}\right)$ and $\left(Y_{x}, \mathcal{G}_{x}\right)$, for $x \in X$, are also ordinal (because a subgeometry of an ordinal geometry is itself ordinal).

To prove the converse, suppose now that $\left(X, \mathcal{G}_{X}\right)$ and $\left(Y_{x}, \mathcal{G}_{x}\right)$, for $x \in X$, are all ordinal convex geometries. By Theorem 5 , it suffices to show that $\mathcal{G}_{Z}$ is closed under union. Let $B, C \in \mathcal{G}_{Z}$. We shall prove that $D=B \cup C$ satisfies Requirements (R1)-(R3) in Definition 19.

(R1) Requirement (R1) applied to $B$ and $C$ yields $\pi(B), \pi(C) \in \mathcal{G}_{X}$, hence $\pi(D)=$ $\pi(B) \cup \pi(C) \in \mathcal{G}_{X}$, because $\mathcal{G}_{X}$ is closed under union.

(R2) Let $x \in \pi(D)$. Without loss of generality, assume that $x \in \pi(B) \cap \pi(D)$ (indeed, if $x$ belongs to exactly one between $\pi(B)$ and $\pi(C)$, the result is trivial). Now Requirement (R2) applied to $B$ and $C$ yields $B \cap Y_{x}, C \cap Y_{x} \in \mathcal{G}_{x}$. Since $D \cap Y_{x}=$ $\left(B \cap Y_{x}\right) \cup\left(C \cap Y_{x}\right)$ and $\mathcal{G}_{x}$ is closed under union by assumption, we derive $D \cap Y_{x} \in \mathcal{G}_{x}$, as claimed.

(R3) Let $x \in \pi(D) \backslash \operatorname{ex}_{\mathcal{G}_{X}}(\pi(D))=(\pi(B) \cup \pi(C)) \backslash \operatorname{ex}_{\mathcal{G}_{X}}(\pi(B) \cup \pi(C))$. It follows that $x \in \pi(B) \backslash \operatorname{ex}_{\mathcal{G}_{X}}(\pi(B))$ or $x \in \pi(C) \backslash \operatorname{ex}_{\mathcal{G}_{X}}(\pi(C))$ holds. By Requirement (R3) applied to $B$ or $C$, we get $Y_{x} \subseteq B$ or $Y_{x} \subseteq C$, hence $Y_{x} \subseteq B \cup C=D$. Thus, $D$ satisfies (R3), too.

Next, we prove the second assertion. Let $\leqslant_{X}, \leqslant_{x}$, and $\leqslant_{Z}$ be the partial orders associated to the ordinal convex geometries $\left(X, \mathcal{G}_{X}\right),\left(Y_{x}, \mathcal{G}_{x}\right)$, and $\left(Z, \mathcal{G}_{Z}\right)$, respectively. 
We show that $\left(Z, \leqslant_{Z}\right)$ is the resolution (in terms of relations) of $\left(X, \leqslant_{X}\right)$ into the family $\left(Y_{x}, \leqslant x\right)_{x \in X}$, that is,

$$
\left(Z, \leqslant_{Z}\right)=\left(X, \leqslant_{X}\right) \square\left(Y_{x}, \leqslant_{x}\right)_{x \in X}
$$

Indeed, in view of the equation (4) in Lemma 23 , the equivalence (16), and the equivalence (17) in Definition 52, we have, for all $z, z^{\prime} \in Z$,

$$
\begin{aligned}
\neg\left(z<_{Z} z^{\prime}\right) \Longleftrightarrow & z \in \operatorname{ex}_{\mathcal{G}_{Z}}\left(\left\{z, z^{\prime}\right\}\right) \\
\Longleftrightarrow & z \in \bigcup_{x \in \operatorname{ex}_{\mathcal{G}_{X}}\left(\pi\left(\left\{z, z^{\prime}\right\}\right)\right)} \operatorname{ex}_{\mathcal{G}_{x}}\left(\left\{z, z^{\prime}\right\} \cap Y_{x}\right) \\
\Longleftrightarrow & (\exists x \in X)\left(z, z^{\prime} \in Y_{x} \wedge z \in \operatorname{ex}_{\mathcal{G}_{x}}\left(\left\{z, z^{\prime}\right\}\right)\right) \text { or } \\
& \left(\exists x, x^{\prime} \in X\right)\left(x \neq x^{\prime} \wedge z \in Y_{x} \wedge z^{\prime} \in Y_{x^{\prime}} \wedge \pi(z) \in \operatorname{ex}_{\mathcal{G}_{X}}\left(\pi\left(\left\{z, z^{\prime}\right\}\right)\right)\right) \\
\Longleftrightarrow & (\exists x \in X)\left(z, z^{\prime} \in Y_{x} \wedge \neg\left(z<_{x} z^{\prime}\right)\right) \text { or } \\
& \left(\exists x, x^{\prime} \in X\right)\left(x \neq x^{\prime} \wedge z \in Y_{x} \wedge z^{\prime} \in Y_{x^{\prime}} \wedge \neg\left(\pi(z)<_{X} \pi\left(z^{\prime}\right)\right)\right) .
\end{aligned}
$$

We conclude that $z<_{Z} z^{\prime}$ does not hold if and only if the pair $\left(z, z^{\prime}\right)$ does not belong to the resolution of the partial order $\leqslant_{X}$ into the family $\left\{\leqslant_{x} \mid x \in X\right\}$. This completes the proof.

The following consequence of Theorem 54 is immediate:

Corollary 55. An ordinal convex geometry is primitive if and only its associated partial order is primitive.

For information on primitive posets, we refer the reader to Schmerl and Trotter (1993) or Boudabbous, Zaguia, and Zaguia (2010). The concept of primitivity applies to more general relational structures: see Ille (2005) for a survey.

The shrinkable sets of an ordinal convex geometry $(Z, \mathcal{G})$ are exactly the autonomous sets of the associated partial order $\leqslant$, where (see Schröder, 2016) $S \subseteq Z$ is autonomous if for all $s, s^{\prime} \in S$ and $z \in Z \backslash S$,

$$
s \leqslant z \quad \Longrightarrow \quad s^{\prime} \leqslant z \quad \text { and } \quad z \leqslant s \quad \Longrightarrow z \leqslant s^{\prime} .
$$

Finally, observe that Theorem 54 does not hold for compositions: indeed, Example 20 exhibits a composition of ordinal convex geometries that fails to be a convex geometry. ${ }^{15}$

\section{$5 \quad$ Primitivity of Small Convex Geometries}

Here we determine all primitive convex geometries on at most four elements. Observe preliminarily that our classification task is simple for the special case of ordinal convex geometries. In fact, by Corollary 55, to test whether an ordinal convex geometry is primitive, it suffices to check whether its associated poset is primitive (as a poset), which in turn amounts to investigate whether the poset has an autonomous subset.

\footnotetext{
${ }^{15} \mathrm{~A}$ close link between compositions of set systems and compositions of posets results from attaching to a poset its set of chains, as explained in Möhring and Radermacher (1984).
} 
To start, note that all convex geometries on one or two elements are primitive. The next proposition inspects which convex geometries on three and four elements are primitive, also determining whether they are ordinal or affine. A list of all 34 convex geometries on four elements appear in Merckx (2013), and their number is confirmed in Uznanski (2013).

Proposition 56. Up to isomorphisms, there are:

(i) 6 convex geometries on three elements, of which 1 is primitive and non-ordinal, and 5 are resolvable and ordinal;

(ii) 34 convex geometries on four elements, 12 of which are primitive; among the primitive ones, 1 is ordinal and 2 are affine.

Proof. (i) On three elements there are, up to isomorphisms, 6 geometries, which are listed in Example 3. By using Theorem 5, one can readily check that exactly 5 of them are ordinal: in fact, there is only one convex geometry that is not closed under union, namely $\mathcal{G}_{5}$. All 5 corresponding posets on three elements are resolvable, hence also the associated ordinal convex geometries are resolvable. Furthermore, the unique non-ordinal geometry $\mathcal{G}_{5}$ is primitive, since otherwise its base and fibers would be ordinal, and so $\mathcal{G}_{5}$ itself would also be ordinal by Theorem 54 .

(ii) On four elements, there are 34 convex geometries, 16 of which are ordinal. The 16 posets on four elements are listed, for instance, in Monteiro, Savini and Viglizzo (2017) and Steinbach (1990); only one of these posets is primitive (it is the 'N-poset'). To find out how many of the 18 non-ordinal convex geometries on four elements are primitive, we rather look for the number of resolvable ones.

By Theorem 54, non-ordinal resolutions have either a non-ordinal base or a non-ordinal fiber (or both). Furthermore, all fibers of a nontrivial resolution on four elements have size at most three. Thus there are only two cases:

(a) the base is the unique non-ordinal geometry $\mathcal{G}_{5}$ on three elements, and the three fibers have one, one, and two elements, respectively;

(b) the base has two elements and one fiber is $\mathcal{G}_{5}$.

Taking into account the automorphisms of small convex geometries, we are left with 7 possible resolutions, of which 4 are of type (a), and 3 of type (b). It is simple to construct these 7 resolvable geometries, and check that they are pairwise non-isomorphic. We conclude that among the 18 non-ordinal convex geometries on four elements, 11 are primitive. Moreover, exactly 2 of these 11 primitive geometries are affine. In fact, there are exactly 4 affine convex geometries on four points, which are those induced on the subsets of the real affine plane shown in Figure 6; only the first and the fourth produce a primitive convex geometry. 

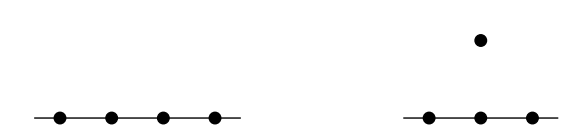

Figure 6: Four subsets of the real affine plane (see the proof of Proposition 56).

\section{$6 \quad$ Future Work and Open Problems}

Here we list a few natural problems (they might be easy or difficult). By a class of convex geometries, we mean a class closed under taking isomorphic images.

1. For a property (P) shared by some convex geometries, consider the following two assertions:

(i) if the base and the fibers of a resolution all satisfy $(\mathrm{P})$, then the resolution also satisfies $(\mathrm{P})$;

(ii) if a resolution satisfies $(\mathrm{P})$, then its base and its fibers satisfy $(\mathrm{P})$.

We say that the property (P) is forward stable when (i) is true, backward stable when (ii) is true, and stable when both (i) and (ii) are true. For instance, ordinality of a convex geometry is a stable property (Theorem 54), whereas affineness is a backward stable property (Lemma 41) that fails to be forward stable (Example 42). An interesting problem consists of determining which (additional) properties of convex geometries considered in the literature are preserved by resolutions, in particular which of the known families of convex geometries are stable under resolutions (see Goecke et al., 1989 for several types of such families). Carpentiere (2019) shows that neither monophonic convex geometries nor geodetic convexity geometries form a stable family (for monophonic vs geodetic convex sets in graphs, see Farber and Jamison, 1986).

2. Any class $\mathcal{C}$ of convex geometries is included in a smallest class $\mathcal{S}$ of convex geometries forward stable under resolutions. When $\mathcal{C}$ itself is not forward stable, $\mathcal{S}$ differs from $\mathcal{C}$. Characterize $\mathcal{S}$ when $\mathcal{C}$ is the class of affine convex geometries, and also for other nonstable classes $\mathcal{C}$.

3. Design a non-naïve algorithm to test whether a given convex geometry is primitive, and another one to generate the primitive convex geometries on small numbers of elements. Enright (2001) investigates various encodings of convex geometries. Uznanski (2013) discusses a code generating all convex geometries up to seven elements (reporting ingegneous programming efforts).

4. As it is the case for many classes of structures with respect to compositions (see Möhring and Radermacher, 1984), does the fraction of primitive convex geometries on $n$ elements tend to one? (Notice that there are two questions here, one for convex geometries on labeled sets and one for convex geometries up to isomorphisms.) About the asymptotic number of labelled convex geometries, see Echenique (2007) and Monjardet 
(2008). For examples of recent results concerning prime structures, see Boudabbous and Ille (2011) for binary relations, Guillet, Leblet, and Rampon (2017) for posets, Ille and Villemaire (2014) as well as Chudnovsky et al. (2016) for graphs.

5. As explained in Section 3, our definition of resolutions of convex geometries was inspired from the one of resolutions of (path-independent) choice spaces in Cantone et al. (2021). On the other hand, Ando (2006) (see also Danilov and Koshevoy, 2009) relate so-called quasi-choice spaces to 'closure spaces'. Investigate (a variant of) resolutions for all those structures.

6. When a convex geometry is expressed as a resolution, the fibers could again be resolvable, and again their fibers, etc. This leads to a notion of 'deresolution tree', which is similar to that of a decomposition tree based on hypergraph compounds (Chein et al., 1981) or set-system compositions (Möhring and Radermacher, 1984; Möhring, 1985). A manuscript under preparation contains a uniqueness result for a well-defined deresolution tree of a convex geometry, and even of a choice space. 'Strong shrinkable sets' are the main tools, similar to strong modules for decomposition trees (for recent work on the latter, see, for instance, Bonizzoni and Della Vedova, 1999; Foldes and Radeleczki, 2016; Habib et al., 2019).

7. Kashiwabara, Nakamura and Okamoto (2005) show that any convex geometry can be obtained by a construction that generalizes the one for affine convex geometries (which is obtained by taking $Q=\varnothing$ below). Specifically, given two finite subsets $P$ and $Q$ in some real affine space $\mathbb{R}^{d}$ with the property that $P \neq \varnothing$ and $P \cap \operatorname{conv}_{\mathbb{R}}(Q)=\varnothing$, let

$$
\mathcal{L}:=\left\{G \in 2^{P} \mid \operatorname{conv}_{\mathbb{R}}(G \cup Q) \cap P=G\right\} .
$$

Then $(P, \mathcal{L})$ is a convex geometry, and moreover any convex geometry is isomorphic to such a geometry. The following are extensions of Problems 47 and 48: (i) characterize the shrinkable subsets of the convex geometry $(P, \mathcal{L})$ in terms of $P$ and $Q$; (ii) characterize the pairs $(P, Q)$ for which the convex geometry $(P, \mathcal{L})$ is primitive.

8. There are some definitions of infinite convex geometries in the literature: see, for instance, Adaricheva (2014b), Adaricheva and Nation (2016d), Jamison-Waldner (1982), Mao (2017), Mao and Liu (2012), Marti and Pinosio (2020), Wahl (2001). In view of these notions, extending the investigation of resolutions to infinite convex geometries appears to be of some interest.

9. As already emphasized in Footnote 7, it may be interesting to study resolutions of special convex geometries, which correspond to choice spaces that are rationalizable by means of 'well-behaved' binary relations - that is, satisfying $(m, n)$-Ferrers properties in the sense of Giarlotta and Watson $(2014,2018)$. This topic of research appears to be closely related to Topic 1 of this list, because several properties of 'choice consistency' are both forward and backward stable: see Cantone et al. (2021). 


\section{Acknowledgments}

The authors wish to thank an anonymous referee for many insightful suggestions and comments. They are also grateful to Davide Carpentiere for several useful discussions on the topic, and Pierre Ille for relevant information on primitive posets.

\section{References}

Adaricheva, K., 2014. Algebraic convex geometries revisited. arXiv:1406.3721

Adaricheva, K., and J. B. Nation, 2016a. Lattices of algebraic subsets and implicational classes. In G. Grätzer and F. Wehrung, eds., Lattice Theory: Special topics and applications, vol.2, ch. 4, pp. 103-151. Birkhäuser/Springer, Cham.

Adaricheva, K., and J.B. Nation, 2016b. Convex geometries. In G. Grätzer and F. Wehrung, eds., Lattice Theory: Special topics and applications, vol. 2, ch. 5, pp. 153-179. Birkhäuser/Springer, Cham.

Adaricheva, K. and J. B. Nation, 2016c. Bases of closure systems. In G. Grätzer and F. Wehrung, eds., Lattice Theory: Special topics and applications, vol. 2, ch.6, pp. 181-213. Birkhäuser/Springer, Cham.

Adaricheva, K., and J. B. Nation, 2016d. A class of infinite convex geometries. The Electronic Journal of Combinatorics 23: \#1.56.

Aizerman, M. A., and A. V. Malishevski, 1981. General theory of best variants choice: some aspects. IEEE Transactions on Automatic Control 26: 1030-1040.

Ando, K., 2006. Extreme point axioms for closure spaces. Discrete Mathematics 306: 3181-3188.

Bang-Jensen, J., and G. Gutin, 2001. Digraphs. Springer Monographs in Mathematics. Springer, London.

Bonizzoni, P., and G. Della Vedova, 1999. An algorithm for the modular decomposition of hypergraphs. Journal of Algorithms 32: 65-86.

Boudabbous, I., and P. Ille. Ultracritical and hypercritical binary structures. Discrete Mathematics 311: 1397-1409.

Boudabbous, Y., I. Zaguia, and N. Zaguia, 2010. Prime orders all of whose prime suborders are selfdual. Order 27: 283-300.

Cantone, D., A. Giarlotta, S. Greco, and S. Watson, 2016. $(m, n)$-rationalizable choices. Journal of Mathematical Psychology 73: 12-27.

Cantone, D., A. Giarlotta, and S. Watson, 2021. Choice resolutions. Social Choice and Welfare 56: $713-753$.

Carpentiere, D., 2019. Resolution of Convex Geometries. Bachelor Thesis, University of Catania, Italy. 
Chernoff, H., 1954. Rational selection of decision functions. Econometrica 22: 422-443.

Chein, M., M. Habib, and M. C. Maurer, 1981. Partitive hypergraphs. Discrete Mathematics 37: $35-50$.

Chudnovsky, M., R. Kim, S.-I. Oum, and P. Seymour. Unavoidable induced subgraphs in large graphs with no homogeneous sets. Journal of Combinatorial Theory, Series B 118: 1-12.

Danilov, V., and G. Koshevoy, 2009. Choice functions and extensive operators. Order 26: 69-94.

Dörfler, W., 1971. Über die X-Summe von gerichteten Graphen. Archiv der Mathematik (Basel), 22: $24-36$.

Doignon, J.-P., and J.-C. Falmagne, 1999. Knowledge Spaces. Springer-Verlag, Berlin.

Echenique, F., 2007. Counting combinatorial choice rules. Games and Economic Behavior 58: $231-245$.

Edelman, P. H., and R. E. Jamison, 1985. The theory of convex geometries. Geometriae Dedicata 19: $247-270$.

Enright, J., 2001 The computational complexity of antimatroid properties. Advances in Applied Mathematics 26: 23-46.

Falmagne J.-C., and J.-P. Doignon, 2011. Learning Spaces. Springer-Verlag, Berlin.

Farber, M., and R.E. Jamison, 1986. Convexity in graphs and hypergraphs. SIAM Journal on Algebraic Discrete Methods 7: 433-444.

Fedorčuk, V.V., 1968. Bicompacta with noncoinciding dimensionalities. Soviet Mathematics Doklady 9: 1148-1150.

Foldes, S., and S. Radeleczki, 2016. Interval decomposition lattices are balanced. Demonstratio Mathematica 49: 271-281.

Giarlotta, A., 2019. New trends in preference, utility, and choice: from a mono-approach to a multi-approach. In M. Doumpos, J. R. Figueira, S. Greco, and C. Zopounidis (eds.), New Perspectives in Multiple Criteria Decision Making, pp. 3-80. Springer, Cham.

Giarlotta, A., and S. Watson, 2014. The pseudo-transitivity of preference relations: strict and weak $(m, n)$-Ferrers properties. Journal of Mathematical Psychology 58: 45-54.

Giarlotta, A., and S. Watson, 2018. Strict $(m, 1)$-Ferrers properties. Journal of Mathematical Psychology 82: 84-96.

Goecke, O., B. Korte, and L. Lovász, 1989. Examples and algorithmic properties of greedoids. In Combinatorial Optimization (Como, 1986), vol.1403 of Lecture Notes in Mathematics, pp. 113-161. Springer, Berlin.

Grätzer, G., 2011. Lattice Theory: Foundation. Birkhäuser/Springer Basel AG, Basel. 
Guillet, A., J. Leblet, and J.-X. Rampon. Faithful extension on finite order classes. The Australasian Journal of Combinatorics 69: 1-17.

Habib, M., F. de Montgolfier, L. Mouatadid, and M. Zou, 2019 A general algorithmic scheme for modular decompositions of hypergraphs and applications. In Combinatorial algorithms, vol. 11638 of Lecture Notes in Computer Science, pp. 251-264. Springer, Cham.

Harzheim, E., 2005. Ordered Sets, vol. 7 of Advances in Mathematics. Springer, New York.

Hiraguchi, T., 1951. On the dimension of partially ordered sets. Science Reports of Kanazawa University 1: 77-94.

Hoffmann, U., and K. Merckx, 2018. A universality theorem for allowable sequences with applications. arXiv: 1801.05992

Ille, P., 2005. La décomposition intervallaire des structures binaires. Gazette des Mathématiciens 104: $39-58$.

Ille, P., and R. Villemaire, 2014. Recognition of prime graphs from a prime subgraph. Discrete Mathematics 327: 76-90.

Jamison-Waldner, R.E., 1982. A perspective on abstract convexity: classifying alignments by varieties. In Convexity and Related Combinatorial Geometry (Norman, Okla., 1980), vol. 76 of Lecture Notes in Pure and Applied Mathematics, pp. 113-150. Dekker, New York.

Johnson, M. and R. Dean, 1996. An algebraic characterization of path independent choice functions. In Third International Meeting of the Society for Social Choice and Welfare, Maastricht, The Netherlands.

Johnson, M. and R. Dean, 2001. Locally complete path independent choice functions and their lattices. Mathematical Social Sciences 42: 53-87.

Kashiwabara, K., M. Nakamura, and Y. Okamoto, 2005. The affine representation theorem for abstract convex geometries. Computational Geometry 30: 129-144.

Korte, B., L. Lovász, and R. Schrader, 1991. Greedoids, vol. 4 of Algorithms and Combinatorics. Springer-Verlag, Berlin.

Koshevoy, G. A., 1999. Choice functions and abstract convex geometries. Mathematical Social Sciences 38: 35-44.

Levi, F.W., 1951. On Helly's theorem and the axioms of convexity. Journal of the Indian Mathematical Society 15(Pt A): 65-76.

Mao, H., 2017. Geometries, independence spaces and infinite antimatroids. Matematika (Johor) 33: $105-111$.

Mao, H., and S. Liu, 2012. On antimatroids of infinite character. Mathematica Pannonica 23: $257-266$. 
Marti, J., and R. Pinosio, 2020. A discrete duality between nonmonotonic consequence relations and convex geometries. Order 37: 151-171.

Méndez, M. A., 2015. Set Operads in Combinatorics and Computer Science. Springer Briefs in Mathematics. Springer, Cham.

Merckx, K., 2013. Descriptions Linéaires de Polytopes Associés aux Antimatroïdes. Master Thesis, Université Libre de Bruxelles, Belgium.

Möhring, R.H., 1985. Algorithmic aspects of the substitution decomposition in optimization over relations, sets systems and Boolean functions. Annals of Operations Research 4: 195-225.

Möhring, R. H., and F. J. Radermacher, 1984. Substitution decomposition for discrete structures and connections with combinatorial optimization. In Algebraic and Combinatorial Methods in Operations Research, vol. 95 of North-Holland Mathematics Studies, pp. 257-355, NorthHolland, Amsterdam.

Monjardet, B., 1985. A use for frequently rediscovering a concept. Order 1: 415-417.

Monjardet, B., 1990. The consequences of Dilworth's work on lattices with unique irreducible decompositions. In The Dilworth Theorems: Selected theorems of Robert P. Dilworth (Contemporary Mathematicians), pp. 192-199. Birkhäuser Boston, MA.

Monjardet, B., 2008. Statement of precedence and a comment on IIA terminology. Games and Economic Behavior, 62: 736-738.

Monjardet, B., and V. Raderanirina, 2001. The duality between the anti-exchange closure operators and the path independent choice operators on a finite set. Mathematical Social Science 41: $131-150$.

Monteiro, L. F., S. Savini, and I. Viglizzo, 2017. Hasse diagrams of non-isomorphic posets with $n$ elements, $2 \leqslant n \leqslant 7$, and the number of posets with 10 elements, without the aid of any computer program. arXiv:1710.10343

Moulin, H., 1985. Choice functions over a finite set: a summary. Social Choice and Welfare 2: $147-160$.

Plott, C.R., 1973. Path independence, rationality, and social choice. Econometrica 41: 10751091.

Samuelson, P., 1938. A note on the pure theory of consumers' behavior. Economica 5: 61-71.

Schmerl, J.H., and W. T. Trotter, 1993. Critically indecomposable partially ordered sets, graphs, tournaments and other binary relational structures. Discrete Mathematics 113: 191-205.

Schröder, B., 2016. Ordered Sets: An introduction with connections from combinatorics to topology. Birkhäuser/Springer, second edition.

Sierksma, G., 1984. Exchange properties of convexity spaces. In M. Rosenfeld and J. Zaks, editors, Annals of Discrete Mathematics (20): Convexity and Graph Theory, vol. 87 of NorthHolland Mathematics Studies, pp. 293-305. North-Holland. 
Steinbach, P., 1990. Field Guide to Simple Graphs. Design Lab, 1990, Albuquerque TechnicalVocational Institute, Albuquerque, NM.

Suzumura, K., 2016. Choice, Preferences, and Procedures: A rational choice theoretic approach. Harvard University Press, Cambridge, MA.

Trotter, W. T., 1992. Combinatorics and Partially Ordered Sets: Dimension theory. The Johns Hopkins University Press, Baltimore, MD.

Uznanski, P., 2013. https://paracombinatorics.wordpress.com/2013/04/19/ enumeration-of-antimatroids-part-iv/ (consulted on October 27, 2020).

Wahl, N., 2001. Antimatroids of finite character. Journal of Geometry 70: 168-175.

Watson, S., 1992. The construction of topological spaces: planks and resolutions. In M. Husek and J. van Mill (eds.), Recent Progress in General Topology, North-Holland, Amsterdam, pp. $673-757$. 